\title{
Iterative Encoder-Controller Design for Feedback Control Over Noisy Channels
}

\author{
Lei Bao, Member, IEEE, Mikael Skoglund, Senior Member, IEEE, and Karl Henrik Johansson, Senior Member, IEEE
}

\begin{abstract}
We study a closed-loop control system with state feedback transmitted over a noisy discrete memoryless channel. With the objective to minimize the expected linear quadratic cost over a finite horizon, we propose a joint design of the sensor measurement quantization, channel error protection, and controller actuation. It is argued that despite that this encoder-controller optimization problem is known to be hard in general, an iterative design procedure can be derived in which the controller is optimized for a fixed encoder, then the encoder is optimized for a fixed controller, etc. Several properties of such a scheme are discussed. For a fixed encoder, we study how to optimize the controller given that full or partial side-information is available at the encoder about the symbols received at the controller. It is shown that the certainty equivalence controller is optimal when the encoder is optimal and has full side-information. For a fixed controller, expressions for the optimal encoder are given and implications are discussed for the special cases when process, sensor, or channel noise is not present. Numerical experiments are carried out to demonstrate the performance obtained by employing the proposed iterative design procedure and to compare it with other relevant schemes.
\end{abstract}

Index Terms-Discrete memoryless channel, joint encoder-controller design, joint source-channel coding, limited information control, quantized feedback control.

\section{INTRODUCTION}

I $\mathrm{N}$ recent years, the demand for sharing resources efficiently in large networked systems has been continuously increasing. However, in many situations, there is a challenging conflict between the amount of transmitted data and the response time. In particular for emerging control applications, not necessarily constrained by today's communication protocols, limits imposed on available signaling bandwidth from communication channels can severely restrict the closed-loop performance and even destabilize the system. Networked control based on limited sensor and actuator information has therefore attracted considerable attention during the past decades.

Manuscript received August 17, 2007; revised June 04, 2009 and December 03, 2009; accepted May 26, 2010. This paper was presented in part at the 2007 American Control Conference and the 2007 IEEE International Symposium on Information Theory. This work was supported in part by the Swedish Research Council, the Swedish Strategic Research Foundation, the Swedish Governmental Agency for Innovation Systems, and the European Commission through the FP7 project FeedNetBack. Recommended by Associate Editor M. Fujita.

The authors are with the ACCESS Linnaeus Center, School of Electrical Engineering, Royal Institute of Technology (KTH), Stockholm SE-10044, Sweden (e-mail: lei.bao@ee.kth.se; mikael.skoglund@ee.kth.se; kallej@ee.kth.se).

Color versions of one or more of the figures in this paper are available online at http://ieeexplore.ieee.org.

Digital Object Identifier 10.1109/TAC.2010.2051833

\section{A. Control Over Data-Rate Limited and Noisy Channels}

Significant research interest has been devoted to the analysis and synthesis of quantized feedback control over a data-rate limited communication link, to stabilize an unstable and noiseless plant. Already in the simplistic situation where the initial state is the only unknown entity that the encoder needs to convey to the controller, a wide range of interesting problems has been formulated. The properties of static quantizers are thoroughly studied in, e.g., [1]-[5]. Advances in ergodic theory and symbolic theory provide new insights into time-invariant and memoryless quantizers. For this type of quantizers, the classical notion of stability is no longer relevant. Instead, attractivity and practical stability are introduced. In [6], the authors present a framework for a quantitative analysis and comparison among different static quantizers. They show the conflicting relation between the steady-state behavior and the contraction rate. In [7], it is proved that a logarithmic quantizer is the coarsest quantizer that quadratically stabilizes a linear time-invariant system. The quantization regions are by most authors assumed to be intervals. In contrast, it is demonstrated in [8] that other quantization sets may have great potential in reducing the transmission rate. Unlike the case of static quantizers, asymptotic stability is possible when time-varying quantizers are used, e.g., [9]-[12]. Asymptotic stability is achieved by employing a "zooming"-strategy in [13]. The basic idea is that the quantizer resolution is increased when the state is close to the equilibrium, while the resolution is reduced when the state is far away.

The fundamental problem of the minimum data rate required to asymptotically stabilize an unstable plant was studied in e.g., [10], [14], assuming a noiseless plant. The relation between the minimum rate and the intrinsic entropy rate of the plant was characterized. Generalizations to more complex situations, e.g., systems with process noise, measurement noise and transmission errors, are challenging research topics. In [15], the authors carried forward the study to systems with process noise and measurement noise. In [16], [17], topological entropy is introduced to study the minimum data rate above which systems with process noise can be stabilized. An excellent survey of the state of the art on rate limited control is given in [18].

In the aforementioned works, the communication channel was essentially assumed to be noise-free, and the only limitation imposed by the channel is the limited data rate. Some recent works on control over a noisy channel include [19]-[30]. For unstable systems, where the allowed delay in the feedback becomes critical, results based on classic Shannon capacity must be used with caution. In particular, with strict delay constraints, the source-channel separation principle may in general not be invoked [31]. Recent work that contributes to updating the classic theories to fit delay-constrained control problems 
includes [19], [24], [32], [37]. These results are typically based on some version of a control-channel separation principle. The basic idea is to design the stabilizing control assuming that the channel is noiseless, and then require the control commands to be reliably transmitted over the noisy channel. In [32], the novel concept of anytime capacity is introduced to show the necessary rate to achieve anytime reliability over noisy channels. This research has been further developed in [19], [23], [38].

Most work on control with limited information has been devoted to stability, while optimal designs are much less explored in the literature. Exceptions include the study of optimal stochastic control over communication channels, e.g., [11], [18], [33]-[36], [39]-[41]. The recent paper [40] provides a useful historical review of linear quadratic Gaussian (LQG) control with quantized feedback. As mentioned in [40], LQG control in the special case of a rate-limited but noiseless feedback link was studied for the first time in the 60'ies, see e.g., [33]-[35]. Later, Fischer [36] characterized the optimal quantizer and controls for this problem in the case of vector-valued state and observation. More recent work includes [39], in which a stable system without measurement noise is considered and an innovation coding scheme is proposed to periodically convey the information about process noise to the controller, over a noisy channel. Since the time between two transmissions is increased when longer codewords are used, there is a trade-off between the resolution and delay. Furthermore, the paper [41] essentially considers the same problem as in [36], that is, quantized control over a rate-limited but noiseless channel, however by restricting the decoder to be memoryless. For this problem, the existence of the optimal controller and its properties are explicitly studied.

A closely related problem to encoder design for quantization of state measurements is the estimation of a Markov source. Some interesting results can be found in [14], [21], [42]. In [14], sequential rate-distortion theory is developed to handle the delay and causality restrictions typically encountered in control systems. In the same paper, the sequential rate-distortion functions for Gaussian-Markov sources are computed. In [21] some theoretical results on real-time (finite decoder memory) encoder-decoders for tracking the outputs of a Markov source are presented.

\section{B. Contributions}

The main contribution of the present paper is the introduction of an iterative design procedure for finding encoder-controller pairs. The result is a synthesis technique for joint optimization of the quantization, error protection and control over a bandlimited and noisy channel. This is an important problem in networked control in the case when a large set of sensor nodes need to limit their individual access to the communication medium. We consider the problem of controlling one single plant in a network where the state measurement is transmitted over a noisy channel. Our system model described in Fig. 1 includes process noise, measurement noise and a finite-rate channel. The details will be specified in Section II. Unlike in most previous work on minimum-data-rate control, our main concern is optimal average performance over a finite horizon, given a fixed data rate. We derive and analyze optimality criteria that we employ to suggest an iterative design algorithm. The performance

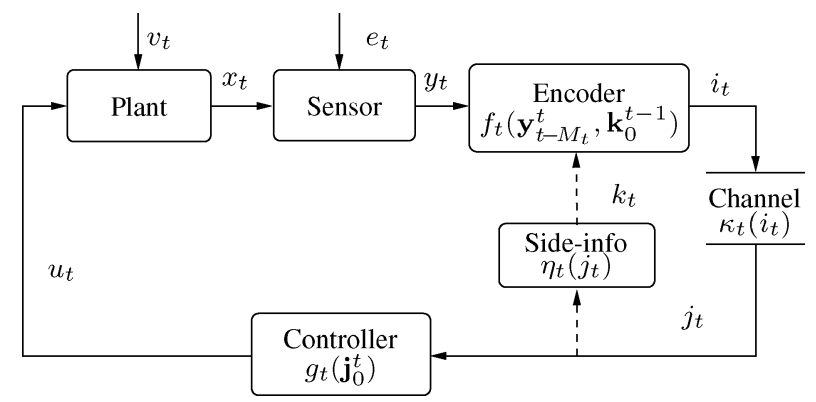

Fig. 1. General system for feedback control over a discrete memoryless channel. The dashed line indicates potential side-information signaling from the controller to the encoder.

of the optimized system is then investigated through numerical simulations.

As a significant step in motivating our optimality criteria, we comment on the validity of decoder-controller separation in iterative encoder-controller design. Similar discussions have previously appeared in, for example, [14], [18], [36], [43]. However, these works focused on existence of optimal systems, while we need a separation criterion that can be used at each step in our design algorithm. As will be discussed below, this leads to some subtle but important differences in the validity of the corresponding results. We also emphasize that, in contrast to [14], [18], [36], we consider control over a noisy channel. Also, in contrast to [36], [43], we allow for general initial state and noise distributions, not necessarily Gaussian; meaning that sufficient statistics at the encoder can in general not be computed based on a Kalman filter, even in the presence of perfect encoder side-information. In addition, we introduce a general model that captures the potential presence of partial side-information at the encoder regarding the received symbols at the controller.

The paper embodies the previous work by the authors on this problem, see [45]-[48].

\section{Organization and Notation}

The paper is organized as follows. In Section II we define the control system with encoder, controller, and communication channel. Thereafter, the problem statement which concerns a linear quadratic (LQ) objective over a finite horizon is formulated. The joint encoder-controller design based on dynamic programming is developed in Sections III and IV. The iterative training algorithm is detailed in Section V. Finally, we present numerical experiments in Section VI and conclusions in the last section.

Some notation used throughout the paper is as follows. Boldfaced characters are used for describing a sequence of signals or functions, e.g., $\mathbf{x}_{a}^{b}=\left\{x_{a}, \ldots, x_{b}\right\}$ denotes the evolution of a discrete-time signal $x_{t}$ from $t=a$ to $t=b$. If $b<a$, then the sequence is empty. We use $\mathbf{E}\{\cdot\}$ to denote the expectation operator, $\operatorname{Tr}(\cdot)$ the trace operator. Also, $p(\cdot)$ denotes a probability density function (pdf) and $P_{r}(\cdot)$ probability. Moreover, the notation $(\cdot)^{\prime}$ stands for matrix transpose and $(\cdot)^{\dagger}$ matrix pseudoinverse. To indicate an optimal solution, the notation $(\cdot)^{*}$ is used.

\section{PRELIMINARIES}

In this section, we first describe the system model in Section II-A. Thereafter we discuss properties of the encoder sideinformation in Section II-B. The performance measure and the 
problem formulation are specified in Section II-C. Finally, in Section II-D, the iterative design is introduced.

\section{A. System Model}

Consider the control system with a communication channel depicted in Fig. 1. Sensor data are encoded and transmitted over an unreliable communication channel. Control commands are derived based on the received data. Next we describe this system in more detail.

The multi-variable linear plant is governed by the following equations:

$$
\begin{aligned}
x_{t+1} & =A x_{t}+B u_{t}+v_{t}, \\
y_{t} & =C x_{t}+e_{t}
\end{aligned}
$$

where $x_{t} \in \mathbb{R}^{n}, u_{t} \in \mathbb{R}^{m}, y_{t} \in \mathbb{R}^{p}$ are the state, the control, and the measurement, respectively. The matrices $A \in \mathbb{R}^{n \times n}$, $B \in \mathbb{R}^{n \times m}, C \in \mathbb{R}^{p \times n}$, are known; moreover, $(A, C)$ is state observable and $(A, B)$ is state controllable. The variable $v_{t} \in$ $\mathbb{R}^{n}$ is the process noise and $e_{t} \in \mathbb{R}^{p}$ is the measurement noise. The noise signals are independent and identically distributed (i.i.d.) and mutually independent. They are also independent of the system initial state $x_{0}$. In addition, we assume that the initial state and the noise processes are zero mean, and their probability density functions are known.

We consider an encoder that causally utilizes the encoder information. By the encoder information, we mean the set of variables whose values are known to the encoder. The encoder is then a mapping from the set of the encoder information to a discrete set of symbols. We take each symbol to be represented by an integer index. At time $t$, the index is $i_{t} \in \mathcal{L}_{i}=\left\{1, \ldots, L_{i}\right\}$, where $L_{i}$ is a fixed integer. In particular, we are interested in the class of encoder mappings described by a function

$$
i_{t}=f_{t}\left(\mathbf{y}_{t-M_{t}}^{t}, \mathbf{k}_{0}^{t-1}\right) .
$$

Here, the parameter $M_{t}$ specifies how many of the past measurements can be used by the encoder. For example, $M_{t}=0$, gives the special case where only the present measurement is available, while when $M_{t}=t$, the encoder can use the full sequence $\mathbf{y}_{0}^{t}$. Given the sequence of past side-information, $\mathbf{k}_{0}^{t-1}$, and the measurements, $\mathbf{y}_{t-M_{t}}^{t}$, the encoder produces an index $i_{t} \in \mathcal{L}_{i}$, and the transmitted index is then received as $j_{t} \in \mathcal{L}_{j}=$ $\left\{1, \ldots, L_{j}\right\}$, where $L_{j} \geq L_{i}$. The side-information $k_{t}$ represents available feedback to the encoder about the value of the symbol $j_{t}$ received at the controller. In this paper, we define the side-information (SI) at the encoder to be produced as

$$
k_{t}=\eta_{t}\left(j_{t}\right) \in \mathcal{L}_{k}=\left\{1, \ldots, L_{k}\right\}, 1 \leq L_{k} \leq L_{j}
$$

where $\eta_{t}: \mathcal{L}_{j} \rightarrow \mathcal{L}_{k}$ is deterministic and memoryless. A detailed discussion on the structure of SI is given in Section II-B.

The encoder output indexes, $i_{t}$, are transmitted over a discrete memoryless channel (DMC), with input and output alphabets $\mathcal{L}_{i}$ and $\mathcal{L}_{j}$, respectively. One use of the channel is defined as

$$
j_{t}=\kappa_{t}\left(i_{t}\right)
$$

where $\kappa_{t}: \mathcal{L}_{i} \rightarrow \mathcal{L}_{j}$ is a random memoryless mapping. Since we assume $L_{j} \geq L_{i}$, the output alphabet is potentially larger than the input alphabet, and hence we allow the possibility of soft information at the channel output. Conditioned on the transmitted symbol $i_{t}$, the mapping to $j_{t}$ is independent of other parameters in the system, e.g., the process and measurement noise. The fact that the channel is bandlimited is captured by the finite size of the input-alphabet $\mathcal{L}_{i}$; we define the rate of the transmission (in bits per channel use) as $R=\log _{2} L_{i}$. Our transmission model encompasses all the common memoryless models; for example, the binary symmetric channel (BSC) and the binary erasure channel (BEC) [49].

At the receiver side, we consider a controller that causally utilizes all the available controller information $\mathbf{j}_{0}^{t}=\left\{j_{s}\right\}_{s=0}^{t}$, to produce the control command

$$
u_{t}=g_{t}\left(\mathbf{j}_{0}^{t}\right) \in \mathbb{R}^{m} .
$$

According to (5), when the sequence $\mathrm{j}_{0}^{t}$ is available at the controller, all past control commands $\mathbf{u}_{0}^{t-1}$ are completely specified.

We denote the conditional mean estimator of the state $x_{s}$, based on the history of the received indexes $\mathbf{j}_{0}^{t}$, as

$$
\hat{x}_{s \mid t}=\mathbf{E}\left\{x_{s} \mid \mathbf{j}_{0}^{t}\right\}, s \leq t .
$$

We will use $\hat{x}_{t}$ as a short notation for $\hat{x}_{t \mid t}=\mathbf{E}\left\{x_{t} \mid \mathbf{j}_{0}^{t}\right\}$. Then, let $\tilde{x}_{t}$ be the estimation error in estimating the state at time $t$, that is, $\tilde{x}_{t}=x_{t}-\hat{x}_{t}=x_{t}-\mathbf{E}\left\{x_{t} \mid \mathbf{j}_{0}^{t}\right\}$.

We note that we will use "encoder" and "controller" in quite general terms ("the corresponding boxes in Fig. 1"), as well as specific terms ("the mappings $f_{t}$ and $g_{t}$ at time $t$ "). When there is a need to be specific, we will adopt the following notation and terminology. Let the notation $\mathbf{f}_{0}^{T-1}=\left\{f_{t}\right\}_{0}^{T-1}$ mean the full sequence of encoder mappings, from time 0 to $T-1$. We will call $\mathrm{f}_{0}^{T-1}$ the "encoder" of the system (since the sequence specifies the operation of the encoder for all relevant time-instants), and we will say that $f_{t}$ is the "encoder mapping at time $t$ " or "the component of $\mathbf{f}_{0}^{T-1}$ at time $t$ " when there is a need to specify a particular time-instant and the corresponding mapping. For subsequences of the full sequence of encoder mappings, we will use similar notation, and say, e.g., "the encoder components $\mathbf{f}_{t_{1}}^{t_{2}}$." This notation will also be used for the controller, its individual mappings or components, and subsequences, say, $\mathbf{g}_{0}^{T-1}, g_{t}$ and $\mathrm{g}_{t_{1}}^{t_{2}}$, respectively.

\section{B. Encoder Side-Information}

Since the transmission rate $R$ is limited, the main motivation for using memory-based encoder-controllers is to increase the resolution of the quantized observation. For memory-based schemes, the system performance relies heavily on the encoder's knowledge about the controller state and the controller's belief in the encoder state. In the presence of a noisy channel, care has to be taken in specifying how to synchronize the states of the encoder and controller. We use the term encoder side-information to specify explicit feedback to the encoder from the controller concerning the received symbols $\mathbf{j}_{0}^{t-1}$. Consequently, no SI is the extreme case when there is no feedback information at all about $j_{t}$, and full SI denotes the situation that the encoder knows exactly the previously received symbols $\mathbf{j}_{0}^{t-1}$. The latter is the case when the channel is noiseless, so that $j_{t}=i_{t}$, or when there is an error-free side-information channel of the same rate from the output of the forward channel to the encoder. 
According to the definition of the side-information mapping $k_{t}=\eta_{t}\left(j_{t}\right)$ in (3), we have $k_{t}=j_{t}$ and $L_{k}=L_{j}$ when full SI is available, while $k_{t}=0$ and $L_{k}=1$ when there is no SI at the encoder. Between the extremes, there are a variety of cases with incomplete SI, for which $1<L_{k}<L_{j}$. Note that in Fig. 1 we illustrate the mapping from $j_{t}$ to $k_{t}$ as an explicit side-information channel, even though this information can be obtained by other means. For example, full SI can be obtained if the encoder knows the previous control commands, $\mathbf{u}_{0}^{t-1}$, and the controller is an invertible mapping, since then $\mathbf{j}_{0}^{t-1}$ can be deduced from $\mathbf{u}_{0}^{t-1}$. In general, when SI is transmitted back to the encoder via a separate noisy channel, assuming the side-information mapping to be deterministic may be optimistic; results based on this assumption then serve as bounds on the achievable performance. However, as we also include the possibility of very low-rate SI feedback (e.g., $L_{k}=2$ ), noiseless transmission may be motivated by the application of heavy error protection in the side-information link.

Via the side-information link the encoder will be informed about the potential transmission errors. However, in contrast to conventional automatic repeat request protocols, no re-transmission will take place. Therefore, the controller must be designed to maintain robustness to transmission errors.

\section{Problem Statement}

Our goal is to solve an optimal encoder-controller problem and thereby finding the suitable encoder and controller mappings. The adopted performance measure is the following LQ cost with finite horizon $T>0$ :

$$
J_{T}=\sum_{t=1}^{T}\left(x_{t}^{\prime} V_{t} x_{t}+u_{t-1}^{\prime} P_{t-1} u_{t-1}\right)
$$

where the matrices $V_{t}$ and $P_{t}$ are symmetric and positive definite. The design objective is to find the encoder-controller mappings which minimize the expected value of the cost $J_{T}$, namely

$$
\mathbf{E}\left\{J_{T}\right\}=\mathbf{E}\left\{\sum_{t=1}^{T} x_{t}^{\prime} V_{t} x_{t}+u_{t-1}^{\prime} P_{t-1} u_{t-1}\right\} .
$$

For ease of reference, we refer to the main design problem as Problem 1, and summarize it below.

Problem 1: Consider the system in Fig. 1. Given the linear plant (1) and the memoryless channel (4), find the encoder (2) and controller (5) that minimize the cost (7).

We use the notation

$$
\left\{f_{t}^{*}\left(\mathbf{y}_{t-M_{t}}^{t}, \mathbf{k}_{0}^{t-1}\right)\right\}_{t=0}^{T-1} \text { and }\left\{g_{t}^{*}\left(\mathbf{j}_{0}^{t}\right)\right\}_{t=0}^{T-1}
$$

for the optimal, but not necessarily unique, mappings that solve Problem 1. Problem 1 can be viewed as an extension of the traditional LQ problem in which the optimal encoder-controller minimizes the cost function with respect to initial state, process noise, measurement noise, and a bandlimited noisy channel.

\section{Iterative Design}

In general, finding an exact solution to Problem 1 is not feasible, because the optimization problem is highly non-linear and non-convex. Therefore, we propose a method to design the encoder-controller pair iteratively, with the goal of finding locally optimal solutions. Inspired by traditional quantizer and vector quantizer designs [50], [51], the idea is to fix the encoder and update the controller, then fix the controller and update the encoder, etc. The iteration terminates when convergence is reached. Unfortunately, the iterative optimization algorithm will not guarantee convergence to a global optimum, but by influencing the initial conditions of the design it is possible to search for good locally optimal designs.

Criteria for updating the encoder and controller are developed in the next two sections. First, the problem of finding the optimal control strategy for a fixed encoder is addressed in Section III. Then, in Section IV, we consider the problem of optimizing one single encoder component, assuming the controller and other encoder components are fixed. The encoding problem for some special cases of the general settings in Section II-A are discussed in more details to illustrate the impact of process noise, measurement noise and transmission errors.

\section{OPtIMAl CONTROLLER FOR FIXED ENCODER}

This section presents the main results of the paper concerning the characterization of optimal controllers. In particular, we investigate the properties of the optimal controller mapping $g_{t}$, assuming the encoder $\mathbf{f}_{0}^{T-1}$ is fixed and given. We begin with a study of the general case in Section III-A, leading to an optimal control equation which is hard to solve. Thereafter, in Section III-B, we investigate the full SI scenario by introducing a modified system using an "open-loop encoder." The optimal control equation is solved for this type of system. Finally, in Section III-C, we discuss how to use the results in Section III-B in designing the system for the general case of partial SI.

\section{A. General Case}

The problem of finding the optimal control assuming the encoder is fixed fits well into the setting of stochastic optimal control, e.g., [52]. We apply dynamic programming to derive the optimal control strategy recursively. In this case, the observations available at the controller are the integer-valued indexes $\mathbf{j}_{0}^{t}$. Resembling a classical result in LQ control, we present the following proposition.

Proposition 1: Consider a fixed encoder $\mathbf{f}_{0}^{T-1}$. Given the plant (1) and the memoryless channel (4), a controller mapping (5) that minimizes the LQ cost (7) fulfills the following recursive relation:

$$
\begin{aligned}
u_{t-1}^{*}= & \arg \min _{u_{t-1}}\left\{\gamma_{t}\right\}, \\
\gamma_{t}= & \lambda_{t}+\mathbf{E}\left\{\gamma_{t+1}^{*} \mid \mathbf{j}_{0}^{t-1}\right\}, \\
\lambda_{t}= & \mathbf{E}\left\{( A x _ { t - 1 } + B u _ { t - 1 } + v _ { t - 1 } ) ^ { \prime } V _ { t } \left(A x_{t-1}\right.\right. \\
& \left.\left.+B u_{t-1}+v_{t-1}\right)+u_{t-1}^{\prime} P_{t-1} u_{t-1} \mid \mathbf{j}_{0}^{t-1}\right\}
\end{aligned}
$$

for $t=1, \ldots, T$, where the cost-to-go $\gamma_{t}$ is initialized with the optimal cost-to-go of $t=T+1$, namely, $\gamma_{T+1}^{*}=0$.

Proof: According to the principle of optimality, e.g., [53], the truncated control sequence $\mathbf{u}_{t}^{* T-1}=\left\{u_{t}^{*}, \ldots, u_{T-1}^{*}\right\}$ is the optimal strategy for the truncated problem corresponding to the 
cost from time $t+1$ to $T$. Hence, the optimal $u_{t}$ is the one that minimizes the sum of the future costs, i.e.

$$
u_{t}^{*}=\arg \min _{u_{t}} \mathbf{E}\left\{\sum_{s=t+1}^{T} x_{s}^{\prime} V_{s} x_{s}+u_{s-1}^{\prime} P_{s-1} u_{s-1} \mid \mathbf{j}_{0}^{t-1}\right\} .
$$

Let us start the recursive derivation at the last stage $t=T$. Since, the optimal cost-to-go at time $T+1$ is zero, i.e., $\gamma_{T+1}^{*}=0$, the optimal $u_{T-1}$ is the one minimizing $\lambda_{T}$, namely

$$
\begin{aligned}
\lambda_{T}= & \mathbf{E}\left\{x_{T}^{\prime} V_{T} x_{T}+u_{T-1}^{\prime} P_{T-1} u_{T-1} \mid \mathbf{j}_{0}^{T-1}\right\}=\operatorname{Tr}\left(V_{T} Q_{T-1}\right) \\
& +\int_{\mathbb{R}^{n}}\left(x_{T-1}^{\prime} A^{\prime} V_{T} A x_{T-1}+2 u_{T-1}^{\prime} B^{\prime} V_{T} A x_{T-1}\right. \\
& \left.\left.+u_{T-1}^{\prime}\left(P_{T-1}+B^{\prime} V_{T} B\right) u_{T-1}\right) P_{r}\left(x_{T-1} \mid \mathbf{j}_{0}^{T-1}\right)\right) d x_{T-1}
\end{aligned}
$$

where the term $Q_{t}=\mathbf{E}\left\{v_{t} v_{t}^{\prime}\right\}$ denotes the covariance matrix of the process noise. Since the noise variance $Q_{T-1}=$ $\mathbf{E}\left\{v_{T-1} v_{T-1}^{\prime}\right\}$ is independent of the control $u_{T-1}$, the control $u_{T-1}$ minimizing $\lambda_{T}$ is then given by

$$
u_{T-1}^{*}=-\left(P_{T-1}+B^{\prime} V_{T} B\right)^{\dagger} B^{\prime} V_{T} A \hat{x}_{T-1}
$$

where $\hat{x}_{T-1}=\mathbf{E}\left\{x_{T-1} \mid \mathbf{j}_{0}^{T-1}\right\}$. Substituting $u_{T-1}^{*}$ into $\lambda_{T}$, the optimal cost-to-go at $t=T$ is

$$
\begin{aligned}
\gamma_{T}^{*} & =\min _{u_{T-1}} \lambda_{T}=\mathbf{E}\left\{x_{T-1}^{\prime} K_{1} x_{T-1}+\varpi_{1} \mid \mathbf{j}_{0}^{T-1}\right\}, \\
K_{1} & =A^{\prime} V_{T} A-\pi_{1}, \\
\pi_{1} & =A^{\prime} V_{T} B\left(P_{T-1}+B^{\prime} V_{T} B\right)^{\dagger} B^{\prime} V_{T} A, \\
\varpi_{1} & =\operatorname{Tr}\left(V_{T} Q_{T-1}\right)+\mathbf{E}\left\{\tilde{x}_{T-1}^{\prime} \pi_{1} \tilde{x}_{T-1} \mid \mathbf{j}_{0}^{T-1}\right\} .
\end{aligned}
$$

At the second last stage $t=T-1$, the optimal $u_{T-2}$ is the one minimizing $\gamma_{T-1}$, as

$$
\begin{aligned}
u_{T-2}^{*} & =\arg \min _{u_{T-2}}\left\{\gamma_{T-1}\right\}, \\
\gamma_{T-1} & =\lambda_{T-1}+\mathbf{E}\left\{\gamma_{T}^{*} \mid \mathbf{j}_{0}^{T-2}\right\}, \\
\lambda_{T-1} & =\mathbf{E}\left\{x_{T-1}^{\prime} V_{T-1} x_{T-1}+u_{T-2}^{\prime} P_{T-2} u_{T-2} \mid \mathbf{j}_{0}^{T-2}\right\} .
\end{aligned}
$$

Generalizing to any time $t$, the optimal control $u_{t-1}^{*}$ can be obtained by solving the following equation:

$$
u_{t-1}^{*}=\arg \min _{u_{t-1}}\left\{\gamma_{t}\right\}=\arg \min _{u_{t-1}}\left\{\lambda_{t}+\mathbf{E}\left\{\gamma_{t+1}^{*} \mid \mathbf{j}_{0}^{t-1}\right\}\right\}
$$

where $\gamma_{t}$ is the cost-to-go, given by

$$
\begin{aligned}
\gamma_{t}= & \lambda_{t}+\mathbf{E}\left\{\gamma_{t+1}^{*} \mid \mathbf{j}_{0}^{t-1}\right\}, \\
\lambda_{t}= & \mathbf{E}\left\{A x_{t-1}+B u_{t-1}+v_{t-1}\right)^{\prime} V_{t} \\
& \left.\times\left(A x_{t-1}+B u_{t-1}+v_{t-1}\right)+u_{t-1}^{\prime} P_{t-1} u_{t-1} \mid \mathbf{j}_{0}^{t-1}\right\} .
\end{aligned}
$$

Note that $\gamma_{0}=\mathbf{E}\left\{J_{T}\right\}$. This concludes the proof.

Unfortunately, it is in general not possible to efficiently solve (12). One main obstruction lies in how the term $\mathbf{E}\left\{\gamma_{t+1}^{*} \mid \mathbf{j}_{0}^{\mathbf{t}}\right\}$ is affected by past controls. Consider for example $t=T-1$. The quantity $\mathbf{E}\left\{\tilde{x}_{T-1}^{\prime} \pi_{1} \tilde{x}_{T-1} \mid \mathbf{j}_{0}^{T-1}\right\}$ in $\gamma_{T}^{*}$ is difficult to analyze, since the received index $j_{T-1}$ is itself a function of $u_{T-2}$ via encoding and transmission. Hence, obtaining an explicit solution to (8) is typically not feasible. In the following two subsections we will first investigate the case of full SI and demonstrate that this assumption significantly simplifies the problem.

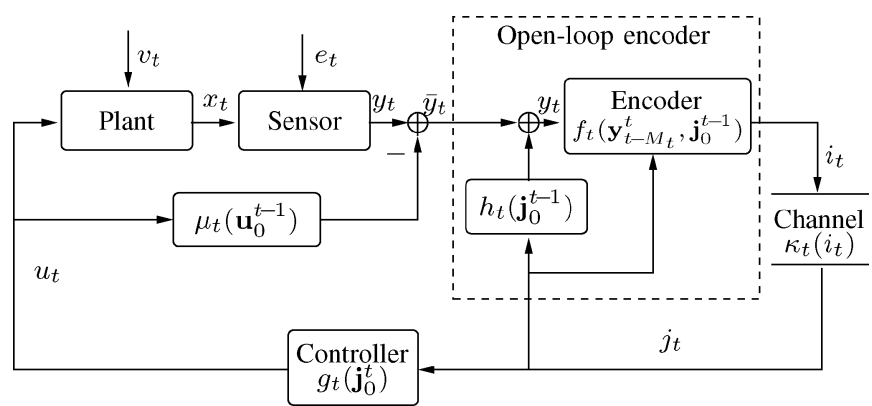

Fig. 2. Equivalent representation of the system.

Then we will discuss how to apply the conclusions derived assuming full SI in the general case.

\section{B. Full Side-Information}

An explicit solution to the optimal control problem (8) can essentially be obtained only in a few special cases. In this section we look at the special case that the encoder has full SI, $k_{t}=$ $j_{t}$. In this case we are able to provide a characterization of the optimal system.

Assume full SI $k_{t}=j_{t}$ is available, that is, the encoder mapping at time $t$ is given as $f_{t}\left(\mathbf{y}_{t-M_{t}}^{t}, \mathbf{j}_{0}^{t-1}\right)$. Now, let us look at the system in Fig. 2, where the function $\mu_{t}\left(\mathbf{u}_{0}^{t-1}\right)$ is the mapping that gives

$$
\bar{y}_{t}=y_{t}-\mu_{t}\left(\mathbf{u}_{0}^{t-1}\right)=y_{t}-\sum_{s=0}^{t-1} C A^{t-1-s} B u_{s} .
$$

Note that $\bar{y}_{t}$ is the part of $y_{t}$ remaining after removing the effect of all previous control commands, so $\bar{y}_{t}$ depends only on $x_{0}$, $\mathbf{v}_{0}^{t-1}$ and $e_{t}$

$$
\bar{y}_{t}=C A^{t} x_{0}+\sum_{s=0}^{t-1} C A^{t-1-s} v_{s}+e_{t} .
$$

That is, $\bar{y}_{t}$ is the equivalent open-loop measurement. Moreover, in Fig. 2 the mapping $h_{t}: \mathcal{L}_{j}^{t} \rightarrow \mathbb{R}^{p}$ is defined as

$$
h_{t}\left(\mathbf{j}_{0}^{t-1}\right)=\sum_{s=0}^{t-1} C A^{t-1-s} B g_{s}\left(\mathbf{j}_{0}^{s}\right)
$$

where the output is used to reproduce the measurement $y_{t}$. Observe that the only parameters needed to specify $\mu_{t}$ are the matrices $A, B$ and $C$, while $h_{t}$ requires knowing the controller mappings $\mathbf{g}_{0}^{t-1}$. That is, if the controller is changed, the mapping $h_{t}$ also changes. Since the operations carried out by $\mu_{t}$ and $h_{t}$ cancel out, it is obvious that the system in Fig. 2 gives exactly the same value for the cost (7) as the one in Fig. 1, provided the same encoder $\mathbf{f}_{0}^{T-1}$ and controller $\mathbf{g}_{0}^{T-1}$ are used.

Now consider the mapping from the open-loop measurement $\bar{y}_{t}$ and the SI $\mathbf{j}_{0}^{t-1}$ to the index $i_{t}$, as enclosed within the dashed line in Fig. 2. We call this the open-loop encoder mapping. Motivated by Fig. 2, we define the system in Fig. 3, assuming the same controller but a completely general mapping $\bar{f}_{t}\left(\overline{\mathbf{y}}_{t-M_{t}}^{t}, \mathbf{j}_{0}^{t-1}\right)$ (that is, one not necessarily related to $f_{t}$ and $h_{t}$ as in Fig. 2). We call the system in Fig. 3 the open-loop encoder system. For a given open-loop encoder system as in Fig. 3, with the open-loop encoder $\overline{\mathbf{f}}_{0}^{T-1}$ and controller $\mathbf{g}_{0}^{T-1}$ specified, one can construct a system with the original structure of Fig. 1 


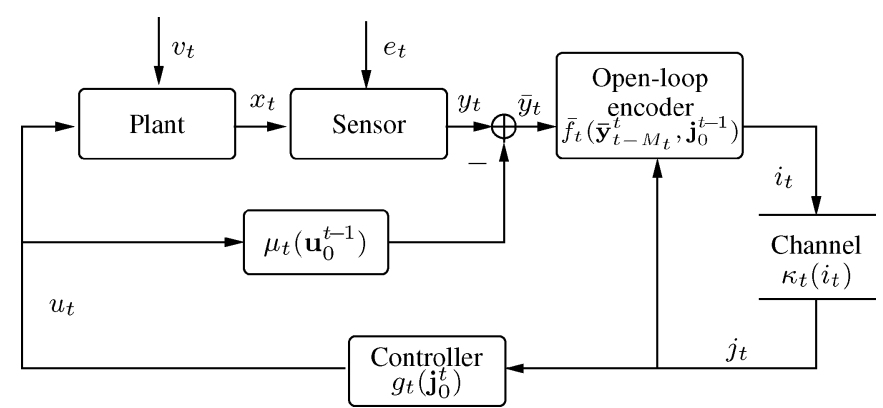

Fig. 3. Open-loop encoder system, a virtual help-system.

which has exactly the same performance. The corresponding system in Fig. 1 utilizes the same controller $\mathbf{g}_{0}^{T-1}$, together with an encoder whose components are determined by $\overline{\mathbf{f}}_{0}^{T-1}$ and $\mathbf{g}_{0}^{T-1}$ as

$$
\begin{aligned}
& f_{t}\left(\mathbf{y}_{t-M_{t}}^{t}, \mathbf{j}_{0}^{t-1}\right) \\
= & \bar{f}_{t}\left(y_{t-M_{t}}-\sum_{s=0}^{t-M_{t}-1} C A^{t-M_{t}-1-s} B g_{s}\left(\mathbf{j}_{0}^{s}\right), \ldots\right. \\
& \left.y_{t}-\sum_{s=0}^{t-1} C A^{t-1-s} B g_{s}\left(\mathbf{j}_{0}^{s}\right), \mathbf{j}_{0}^{t-1}\right), t=0, \ldots, T-1 .
\end{aligned}
$$

For the open-loop encoder system, we formulate the following problem.

Problem 2: Consider an open-loop encoder system as illustrated in Fig. 3. Given the linear plant (1) and the memoryless channel (4), find the open-loop encoder and controller mappings $\left\{\bar{f}_{t}^{*}\left(\overline{\mathbf{y}}_{t-M_{t}}^{t}, \mathbf{j}_{0}^{t-1}\right)\right\}_{t=0}^{T-1}$ and $\left\{g_{t}^{*}\left(\mathbf{j}_{0}^{t-1}\right)\right\}_{t=0}^{T-1}$ that minimize the cost (7).

It is worthwhile here to emphasize our line-of-thought: Neither of the systems in Figs. 2-3 fits our original model in Fig. 1, in particular since in Fig. 1 there is no connection from the controls to the encoder (via the mapping $\mu_{t}$ ). Hence it is not obvious why formulating Problem 2 is relevant. In the following propositions we will however demonstrate how the optimal control problem can be solved for the open-loop encoder system in Fig. 3, while we are not able to solve it in the case of the original system in Fig. 1. We therefore emphasize that the purpose of introducing the open-loop encoder system in Fig. 3 is to have access to a "virtual help-system" in the sense that we will optimize the design for this system and argue that the solution is useful also in the original system.

Open-loop systems similar to the one in Fig. 3 were considered also in [14], [18] (for noiseless channels). However, there is a very important, albeit quite subtle, difference in that the equivalent systems in [14], [18] need to use the controller mapping inside the encoder to "open the loop." This is in contrast to our open-loop encoder system in Fig. 3 where the encoder is a completely arbitrary mapping, and where the loop is opened by subtracting the controls via $\mu_{t}$. This is crucial for the approach in this paper, since in each step of the iterative design the controller is fixed while updating the encoder, and vice versa. In particular, a controller that perfectly fits the encoder is not known when updating the encoder. Hence, as will be demonstrated, our open-loop encoder system is useful in iterative design, while this is not the case with previously proposed equivalent systems. To our knowledge, this issue has not been settled in previous work.
Now, given the plant, the memoryless channel and the design criterion, the solutions to the original Problem 1 and the corresponding Problem 2 for the open-loop encoder system are closely related, as revealed by the following proposition.

Proposition 2:

I. Consider a solution $\left\{f_{t}^{*}\left(\mathbf{y}_{t-M_{t}}^{t}, \mathbf{j}_{0}^{t-1}\right), g_{t}^{*}\right\}_{t=0}^{T-1}$ to Problem 1. The same controller $\mathbf{g}_{0}^{* T-1}$ and the open-loop encoder specified by $\left\{f_{t}^{*}\left(\mathbf{y}_{t-M_{t}}^{t}, \mathbf{j}_{0}^{t-1}\right), g_{t}^{*}\right\}_{t=0}^{T-1}$ as

$$
\begin{aligned}
& \bar{f}_{t}\left(\overline{\mathbf{y}}_{t-M_{t}}^{t}, \mathbf{j}_{0}^{t-1}\right) \\
= & f_{t}^{*}\left(\bar{y}_{t-M_{t}}+\sum_{s=0}^{t-M_{t}-1} C A^{t-M_{t}-1-s} B g_{s}^{*}\left(\mathbf{j}_{0}^{s}\right), \ldots,\right. \\
& \left.\bar{y}_{t}+\sum_{s=0}^{t-1} C A^{t-1-s} B g_{s}^{*}\left(\mathbf{j}_{0}^{s}\right), \mathbf{j}_{0}^{t-1}\right), t=0, \ldots, T-1
\end{aligned}
$$

jointly solve Problem 2.

II. Consider a solution $\left\{\bar{f}_{t}^{*}\left(\overline{\mathbf{y}}_{t-M_{t}}^{t}, \mathbf{j}_{0}^{t-1}\right), g_{t}^{*}\right\}_{t=0}^{T-1}$ to Problem 2. The same controller $\mathrm{g}_{0}^{* T-1}$ and the encoder $\left\{f_{t}\left(\mathbf{y}_{t-M_{t}}^{t}, \mathbf{j}_{0}^{t-1}\right)\right\}_{t=0}^{T-1}$ specified by $\left\{\bar{f}_{t}^{*}\left(\overline{\mathbf{y}}_{t-M_{t}}^{t}, \mathbf{j}_{0}^{t-1}\right), g_{t}^{*}\right\}_{t=0}^{T-1}$ according to

$$
\begin{aligned}
& f_{t}\left(\mathbf{y}_{t-M_{t}}^{t}, \mathbf{j}_{0}^{t-1}\right) \\
= & \bar{f}_{t}^{*}\left(y_{t-M_{t}}-\sum_{s=0}^{t-M_{t}-1} C A^{t-M_{t}-1-s} B g_{s}^{*}\left(\mathbf{j}_{0}^{s}\right), \ldots,\right. \\
& \left.y_{t}-\sum_{s=0}^{t-1} C A^{t-1-s} B g_{s}^{*}\left(\mathbf{j}_{0}^{s}\right), \mathbf{j}_{0}^{t-1}\right), t=0, \ldots, T-1
\end{aligned}
$$

jointly solve Problem 1.

Proof:

I. If the open-loop encoder $\left\{\bar{f}_{t}\left(\overline{\mathbf{y}}_{t-M_{t}}^{t}, \mathbf{j}_{0}^{t-1}\right)\right\}_{t=0}^{T-1}$ according to (14), and $\mathrm{g}_{0}^{* T-1}$ do not jointly solve Problem 2, then another solution to Problem 2 provides a cost lower than the one given by $\left\{\bar{f}_{t}\left(\overline{\mathbf{y}}_{t-M_{t}}^{t}, \mathbf{j}_{0}^{t-1}\right), g_{t}^{*}\right\}_{t=0}^{T-1}$. If this is the case, using the encoder specified by the solution to Problem 2 according to (13), jointly with the controller of the same solution, must lead to a lower cost than the one resulting from $\left\{f_{t}^{*}\left(\mathbf{y}_{t-M_{t}}^{t}, \mathbf{j}_{0}^{t-1}\right), g_{t}^{*}\right\}_{t=0}^{T-1}$. This contradicts the statement that $\left\{f_{t}^{*}\left(\mathbf{y}_{t-M_{t}}^{t}, \mathbf{j}_{0}^{t-1}\right), g_{t}^{*}\right\}_{t=0}^{T-1}$ is a solution to Problem 1. Hence, the statement in Proposition 2 must be true.

II. The proof is similar to part I.

Proposition 2 indicates that a solution to Problem 2 specifies a solution to Problem 1, and vice versa. As we will discuss below, when using the iterative design approach, Problem 2 is in general easier to solve than Problem 1. Hence, in the special case of full SI we will focus on finding a solution to Problem 2, and then derive a corresponding solution to Problem 1 according to (15). Again, we note that Problem 2 is not an equivalent problem (as the system in Fig. 1 cannot be transformed into the system in Fig. 3). However, we will argue that striving to solve Problem 2 will result in encoder-controller pairs that can be converted to fit the original scenario in Fig. 1. Recall that the iterative design approach alternates between specifying an encoder for a fixed controller and updating the controller for a fixed encoder. In the remaining part of this subsection, we will therefore study the 
optimal control problem for the open-loop encoder system in Fig. 3, assuming a fixed and general open-loop encoder $\overline{\mathbf{f}}_{0}^{T-1}$ (this encoder may be completely unrelated to any encoder $\mathbf{f}_{0}^{T-1}$ in the original system).

Consider now a fixed sequence of open-loop encoder mappings $\overline{\mathbf{f}}_{0}^{T-1}$ in the open-loop encoder system (that is, $\bar{f}_{t}$ is fixed and does not change when the controller mappings change). Note that for any such fixed mappings, the transmitted indexes $\mathbf{i}_{0}^{t}$ do not depend on the controls $\mathbf{u}_{0}^{t-1}$, since the open-loop measurements $\overline{\mathbf{y}}_{t-M_{t}}^{t}$ does not depend on $\mathbf{u}_{0}^{t-1}$ and since $i_{0}=$ $\bar{f}_{0}\left(\bar{y}_{0}\right), i_{1}=\bar{f}_{1}\left(\overline{\mathbf{y}}_{1-M_{1}}^{1}, j_{0}\right), i_{2}=\bar{f}_{2}\left(\overline{\mathbf{y}}_{2-M_{2}}^{2}, j_{0}, j_{1}\right)$, etc., and $j_{t}$ depends only on $i_{t}$ and potential channel errors. Because of this fact, we will be able to solve (8), as revealed by the proof of the following proposition.

Proposition 3: Consider the open-loop encoder system in Fig. 3, assuming a fixed open-loop encoder $\overline{\mathbf{f}}_{0}^{T-1}=$ $\left\{\bar{f}_{t}\left(\overline{\mathbf{y}}_{t-M_{t}}^{t}, \mathbf{j}_{0}^{t-1}\right)\right\}_{t=0}^{T-1}$. Given the plant (1) and the memoryless channel (4), the controller component $u_{t}=g_{t}\left(\mathbf{j}_{0}^{t}\right)$ that minimizes the LQ cost (7) is given by

$$
u_{t}=\ell_{t} \hat{x}_{t}
$$

where $\hat{x}_{t}=\mathbf{E}\left\{x_{t} \mid \mathbf{j}_{0}^{t}\right\}$. The control gain $\ell_{t}$ can be recursively computed as

$$
\begin{aligned}
\ell_{t}= & -\left(P_{t}+B^{\prime}\left(V_{t+1}+K_{T-t-1}\right) B\right)^{\dagger} \\
& \times B^{\prime}\left(V_{t+1}+K_{T-t-1}\right) A \\
K_{T-t-1}= & A^{\prime}\left(V_{t+2}+K_{T-t-2}\right) A-\pi_{T-t-1}, \\
\pi_{T-t-1}= & A^{\prime}\left(V_{t+2}+K_{T-t-2}\right) B\left(P_{t+1}+B^{\prime}\left(V_{t+2}\right.\right. \\
& \left.\left.+K_{T-t-2}\right) B\right)^{\dagger} B^{\prime}\left(V_{t+2}+K_{T-t-2}\right) A
\end{aligned}
$$

where $K_{t}$ is initialized with $K_{1}=A^{\prime} V_{T} A-A^{\prime} V_{T} B\left(P_{T-1}+\right.$ $\left.B^{\prime} V_{T} B\right)^{\dagger} B^{\prime} V_{T} A$.

Proof: According to Fig. 3, the open-loop encoder has access to the open-loop measurement $\overline{\mathbf{y}}_{t-M_{t}}^{t}$ and $\mathbf{j}_{0}^{t-1}$. When the sequence of mappings $\overline{\mathbf{f}}_{0}^{T-1}$ is fixed, we can verify that the estimation error $\tilde{x}_{t}$ is not a function of $\mathbf{u}_{0}^{t-1}$ as follows:

$$
\tilde{x}_{t}=A^{t} x_{0}+\sum_{s=0}^{t-1} A^{t-1-s} v_{s}-\mathbf{E}\left\{A^{t} x_{0}+\sum_{s=0}^{t-1} A^{t-1-s} v_{s} \mid \mathbf{j}_{0}^{t}\right\}
$$

since the controls $\mathbf{u}_{0}^{t-1}$ are completely determined by the received symbols $\mathbf{j}_{0}^{t-1}$. As $\left\{x_{0}, \mathbf{v}_{0}^{T-1}, \mathbf{e}_{0}^{T-1}\right\}$ are not affected by $\mathbf{u}_{0}^{T-1}$, one can show by the following induction that the indexes $\mathbf{j}_{0}^{t}$ are not functions of $\mathbf{u}_{0}^{t-1}$. Start the induction at $t=0$. The statement holds true at $t=0$ since

$$
i_{0}=\bar{f}_{0}\left(\bar{y}_{0}\right)=\bar{f}_{0}\left(C x_{0}+e_{0}\right), j_{0}=\kappa_{0}\left(i_{0}\right), k_{0}=j_{0} .
$$

Assuming for the moment the statement is valid for time $t$, as already validated for $t=0$, then at time $t+1$

$i_{t+1}=\bar{f}_{t+1}\left(\overline{\mathbf{y}}_{t+1-M_{t}}^{t+1}, \mathbf{j}_{0}^{t}\right), \quad j_{t+1}=\kappa_{t+1}\left(i_{t+1}\right), \quad k_{t+1}=j_{t+1}$

which hence do not involve $\mathbf{u}_{0}^{t}$. Therefore, $\mathbf{i}_{0}^{T-1}$ and $\mathbf{j}_{0}^{T-1}$ depend only on $\left\{x_{0}, \mathbf{v}_{0}^{T-1}, \mathbf{e}_{0}^{T-1}\right\}$ and potential channel errors, but not on $\mathbf{u}_{0}^{T-1}$.

The fact that the estimation error $\tilde{x}_{t}=x_{t}-\hat{x}_{t}$ is not a function of $\mathbf{u}_{0}^{t-1}$ for the fixed mappings $\left\{\bar{f}_{t}\left(\overline{\mathbf{y}}_{t-M_{t}}^{t}, \mathbf{j}_{0}^{t-1}\right)\right\}_{t=0}^{T-1}$ will significantly simplify the derivation of the optimal control. According to previous calculations, the optimal $u_{T-1}$ is given by
(9), irrespective of the encoding. In order to derive the optimal $u_{T-2}$, let us consider (10) and (11). Since the covariance of the estimation error $\tilde{x}_{T-1}$ is independent of $\mathbf{u}_{0}^{T-2}$, we can find the optimal $u_{T-2}$ as

$$
\begin{aligned}
& u_{T-2}^{*}=\ell_{T-2} \hat{x}_{T-2}, \\
& \ell_{T-2}=-\left(P_{T-2}+B^{\prime}\left(V_{T-1}+K_{1}\right) B\right)^{\dagger} B^{\prime}\left(V_{T-1}+K_{1}\right) A
\end{aligned}
$$

and the optimal cost-to-go at $t=T-1$ can be written as

$$
\begin{aligned}
\gamma_{T-1}^{*}= & \mathbf{E}\left\{x_{T-2}^{\prime} K_{2} x_{T-2}+\varpi_{2} \mid \mathbf{j}_{0}^{T-2}\right\}, \\
K_{2}= & A^{\prime}\left(V_{T-1}+K_{1}\right) A-\pi_{2}, \\
\pi_{2}= & A^{\prime}\left(V_{T-1}+K_{1}\right) B\left(P_{T-2}+B^{\prime}\left(V_{T-1}+K_{1}\right) B\right)^{\dagger} \\
& \times B^{\prime}\left(V_{T-1}+K_{1}\right) A, \\
\varpi_{2}= & \varpi_{1}+\operatorname{Tr}\left(\left(V_{T-1}+K_{1}\right) Q_{T-2}\right) \\
& +\mathbf{E}\left\{\tilde{x}_{T-2}^{\prime} \pi_{2} \tilde{x}_{T-2} \mid \mathbf{j}_{0}^{T-2}\right\}
\end{aligned}
$$

with $\left\{K_{1}, \pi_{1}, \varpi_{1}\right\}$ as given in (10).

Assume $u_{t+1}^{*}$ for time $t+1$ is established, as already validated for $t=T-1$, let us continue the derivation of $u_{t}^{*}$. According to Proposition 1, the optimal control $u_{t}^{*}$ is the one solving the following equation:

$$
u_{t}^{*}=\arg \min _{u_{t}}\left\{\lambda_{t+1}+\mathbf{E}\left\{\gamma_{t+2}^{*} \mid \mathbf{j}_{0}^{t}\right\}\right\}
$$

where $\lambda_{t+1}=\mathbf{E}\left\{x_{t+1}^{\prime} V_{t+1} x_{t+1}+u_{t}^{\prime} P_{t} u_{t} \mid \mathbf{j}_{0}^{t}\right\}$. By (18), $\gamma_{t+2}^{*}$ can be written as

$$
\begin{aligned}
\gamma_{t+2}^{*}= & \mathbf{E}\left\{x_{t+1}^{\prime} K_{T-t-1} x_{t+1}+\varpi_{T-t-1} \mid \mathbf{j}_{0}^{t+1}\right\}, \\
\varpi_{T-t-1}= & \varpi_{T-t-2}+\operatorname{Tr}\left(\left(V_{t+2}+K_{T-t-2}\right) Q_{t+1}\right) \\
& +\mathbf{E}\left\{\tilde{x}_{t+1}^{\prime} \pi_{T-t-1} \tilde{x}_{t+1} \mid \mathbf{j}_{0}^{t+1}\right\} .
\end{aligned}
$$

Then, since $\mathbf{E}\left\{\tilde{x}_{t+1}^{\prime} \pi_{T-t-1} \tilde{x}_{t+1} \mid \mathbf{j}_{0}^{t}\right\}$ does not depend on $\mathbf{u}_{0}^{t-1}$ at any time instance $t$, we can obtain the optimal $u_{t}$ by solving the following equation:

$$
\begin{aligned}
u_{t}^{*} & =\arg \min _{u_{t}}\left\{\mathbf{E}\left\{x_{t+1}^{\prime} V_{t+1} x_{t+1}+u_{t}^{\prime} P_{t} u_{t} \mid \mathbf{j}_{0}^{t}\right\}\right. \\
& \left.+\mathbf{E}\left\{x_{t+1}^{\prime} K_{T-t-1} x_{t+1} \mid \mathbf{j}_{0}^{t}\right\}\right\}
\end{aligned}
$$

and the solution is given by

$$
\begin{aligned}
u_{t}^{*} & =\ell_{t} \hat{x}_{t}, \\
\ell_{t} & =-\left(P_{t}+B^{\prime}\left(V_{t+1}+K_{T-t-1}\right) B\right)^{\dagger} B^{\prime}\left(V_{t+1}+K_{T-t-1}\right) A .
\end{aligned}
$$

Finally, the resulting optimal cost-to-go $\gamma_{t+1}^{*}$ is obtained by replacing $u_{t}$ in $\gamma_{t+1}$ with $u_{t}^{*}$

$$
\begin{aligned}
\gamma_{t+1}^{*}= & \mathbf{E}\left\{x_{t}^{\prime} K_{T-t} x_{t}+\varpi_{T-t} \mid \mathbf{j}_{0}^{t}\right\}, \\
K_{T-t}= & A^{\prime}\left(V_{t+1}+K_{T-t-1}\right) A-\pi_{T-t}, \\
\pi_{T-t}= & A^{\prime}\left(V_{t+1}+K_{T-t-1}\right) B\left(P_{t}+B^{\prime}\left(V_{t+1}+K_{T-t-1}\right) B\right)^{\dagger} \\
& \times B^{\prime}\left(V_{t+1}+K_{T-t-1}\right) A, \\
\varpi_{T-t}= & \varpi_{T-t-1}+\operatorname{Tr}\left(\left(V_{t+1}+K_{T-t-1}\right) Q_{t}\right) \\
& +\mathbf{E}\left\{\tilde{x}_{t}^{\prime} \pi_{T-t} \tilde{x}_{t} \mid \mathbf{j}_{0}^{t}\right\} .
\end{aligned}
$$

The results in (16) and (17) illustrate that given a fixed openloop encoder $\overline{\mathbf{f}}_{0}^{T-1}$, it is possible to explicitly characterize the 
optimal control strategy (8). Observe that the optimal control strategy (16) can be decomposed into a separate estimator/decoder and a controller. Hence, the separation property holds, e.g., [52], [54]. Additionally, one can show that the derived optimal control strategy (16) is a certainty equivalence (CE) controller. A CE controller is obtained by first computing the optimal deterministic control, in the absence of process noise and assuming that perfect state observations are available at the controller. Thereafter, the perfect state observations are replaced with estimates of the partially observed states, cf., (16). The CE controller does in general not provide optimum performance. In our case, we are able to show that the resulting CE controller in (16) is optimal for the open-loop encoder system in Fig. 3, assuming a fixed open-loop encoder. However, since the open-loop encoder system is not an instance of the original system in Fig. 1, we cannot claim that the CE controller structure is optimal given a fixed encoder $\mathbf{f}_{0}^{T-1}$ in the original system.

The CE controller is optimal if the second moment of the estimation error $\tilde{x}_{t}$ does not depend on past controls [14], [55]. This is obviously the case in the open-loop system since the dependence on $u_{t}$ is removed before encoding. (This is not needed in the classical LQG problem, where the CE controller is optimal because the estimation error is Gaussian distributed with a fixed variance.) As mentioned, similar approaches are also exploited in, e.g., [14], [18], assuming fully observed plants and noiseless transmission. The problem gets more involved when measurement noise and transmission errors are present. And, again, the corresponding open-loop system in [18] requires the encoder to have instantaneous access to the controller mapping, while our result is directly applicable to iterative encoder-controller design (as described in Section V).

Given a fixed encoder $\mathbf{f}_{0}^{T-1}$, used in the original system in Fig. 1, the CE controller is not necessarily the corresponding optimal control strategy. Still, in the jointly optimal pair $\left\{\mathbf{f}_{0}^{* T-1}, \mathbf{g}_{0}^{* T-1}\right\}$ that solves Problem 1, the controller $\mathbf{g}_{0}^{* T-1}$ is a CE controller, as concluded in the following proposition.

Proposition 4: If $\left\{f_{t}^{*}\left(\mathbf{y}_{t-M_{t}}^{t}, \mathbf{j}_{0}^{t-1}\right), g_{t}^{*}\right\}_{t=0}^{T-1}$ solves Problem 1, then the controller $\mathrm{g}^{* T-1}$ is the CE controller given by (16)-(17) for $\mathbf{f}_{0}^{T-1}=\mathbf{f}_{0}^{* T-1}$.

Proof: Given the linear plant (1), the memoryless channel (4) and the cost function (7), one can find a solution $\left\{\bar{f}_{t}^{*}\left(\mathbf{y}_{t-M_{t}}^{t}, \mathbf{j}_{0}^{t-1}\right), g_{t}^{*}\right\}_{t=0}^{T-1}$ to Problem 2. Then, according to Proposition 2, the following encoder:

$$
\begin{aligned}
& f_{t}^{*}\left(\mathbf{y}_{t-M_{t}}^{t}, \mathbf{j}_{0}^{t-1}\right) \\
& =\bar{f}_{t}^{*}\left(y_{t-M_{t}}-\sum_{s=0}^{t-M_{t}-1} C A^{t-M_{t}-1-s} B g_{s}^{*}\left(\mathbf{j}_{0}^{s}\right), \ldots,\right. \\
& \left.\quad y_{t}-\sum_{s=0}^{t-1} C A^{t-1-s} B g_{s}^{*}\left(\mathbf{j}_{0}^{s}\right), \mathbf{j}_{0}^{t-1}\right), t=0, \ldots, T-1
\end{aligned}
$$

and the controller $\mathrm{g}_{0}^{* T-1}$ jointly specify a solution to Problem 1. As shown by Proposition $3, \mathrm{~g}_{0}^{* T-1}$ given by (16)-(17), is a CE controller in the open-loop encoder system. Observe that given $\overline{\mathbf{y}}_{t-M_{t}}^{t}$ and $\mathbf{j}_{0}^{t-1}, \bar{f}_{t}^{*}\left(\overline{\mathbf{y}}_{t-M_{t}}^{t}, \mathbf{j}_{0}^{t-1}\right)$ and $f_{t}^{*}\left(\mathbf{y}_{t-M_{t}}^{t}, \mathbf{j}_{0}^{t-1}\right)$ produce exactly the same $i_{t}$. Therefore, $\hat{x}_{t}$ and consequently the CE controller are identical for both systems in Problem 1 and Problem 2.
Again, Proposition 4 states that the optimal controller corresponding to the optimal encoder $\mathrm{f}_{0}^{* T^{-1}}$ is the CE controller. While, in the general case, the optimal controller corresponding to any encoder $\mathbf{f}_{0}^{T-1}$ does not necessarily satisfy the separation principle. This is in contrast to the open-loop encoder system, where the optimal controller for any given open-loop encoder is a CE controller. Thus, in designing for the "virtual help-system" defined by the open-loop encoder system, we can use a CE controller in each step of the design. Then, since we know that the controller in the optimal pair for the original system can be separated, we employ the design we get for the open-loop system in the original system, via translation. Proposition 4 motivates this last step.

\section{Partial Side-Information}

When only partial SI $\left(L_{k}<L_{j}\right)$ is available at the encoder in the case of the original system in Fig. 1, there is no result corresponding to Proposition 4 that motivates using the open-loop encoder system with a CE controller as a basis for the design. In the optimal encoder-controller pair for the original system, the controller may not be separated without loss in the case of partial SI. Since we are not able to solve (8) in the general case, we resort to using the $\mathrm{CE}$ controller as a sub-optimal alternative to solving (8). That is, in the case of partial SI at the encoder, we propose an iterative design for the original system in Fig. 1 based on constraining the controller to be a CE controller. For completeness, and for later reference, we state the corresponding expressions as follows: Consider the original system Fig. 1, assuming a fixed encoder $\mathbf{f}_{0}^{T-1}$. By the "CE controller" for this system, we mean the corresponding mapping

$$
u_{t}=\ell_{t} \hat{x}_{t}
$$

where $\hat{x}_{t}=\mathbf{E}\left\{x_{t} \mid \mathbf{j}_{0}^{t}\right\}$, and where the control gain $\ell_{t}$ is computed as

$$
\begin{aligned}
\ell_{t}= & -\left(P_{t}+B^{\prime}\left(V_{t+1}+K_{T-t-1}\right) B\right)^{\dagger} \\
& \times B^{\prime}\left(V_{t+1}+K_{T-t-1}\right) A \\
K_{T-t-1}= & A^{\prime}\left(V_{t+2}+K_{T-t-2}\right) A-\pi_{T-t-1}, \\
\pi_{T-t-1}= & A^{\prime}\left(V_{t+2}+K_{T-t-2}\right) B\left(P_{t+1}+B^{\prime}\left(V_{t+2}\right.\right. \\
& \left.\left.+K_{T-t-2}\right) B\right)^{\dagger} B^{\prime}\left(V_{t+2}+K_{T-t-2}\right) A
\end{aligned}
$$

where $K_{t}$ is initialized as $K_{1}=A^{\prime} V_{T} A-A^{\prime} V_{T} B\left(P_{T-1}+\right.$ $\left.B^{\prime} V_{T} B\right)^{\dagger} B^{\prime} V_{T} A$.

\section{OPTIMAL ENCODER FOR FIXED CONTROLLER}

In this section, we address the problem of optimizing the encoder component $f_{t}$, for a fixed controller $\mathrm{g}_{0}^{T-1}$ and fixed encoder components $\mathbf{f}_{0}^{t-1}, \mathbf{f}_{t+1}^{T-1}$. The optimal encoder mapping needs to take the impact of the predicted future state evolutions into account. The following results are a straightforward consequence of the system assumptions and the design criterion.

Proposition 5: Consider a fixed controller $\mathbf{g}_{0}^{T-1}$ and fixed encoder components $\mathbf{f}_{0}^{t-1}, \mathbf{f}_{t+1}^{T-1}$. Given the linear plant (1) and the memoryless channel (4), the encoder component $f_{t}\left(\mathbf{y}_{t-M_{t}}^{t}, \mathbf{k}_{0}^{t-1}\right)$ that minimizes the LQ cost (7) is given by

$$
\begin{aligned}
i_{t}= & \arg \min _{i \in \mathcal{L}_{i}} \mathbf{E}\left\{\sum _ { s = t + 1 } ^ { T } \left(x_{s}^{\prime} V_{s} x_{s}\right.\right. \\
& \left.\left.+u_{s-1}^{\prime} P_{s-1} u_{s-1}\right) \mid \mathbf{y}_{t-M_{t}}^{t}, \mathbf{k}_{0}^{t-1}, i_{t}=i\right\} .
\end{aligned}
$$


Proof: The proof follows the principle of optimality. Recall the average LQ cost criterion:

$$
\mathbf{E}\left\{J_{T}\right\}=\mathbf{E}\left\{\sum_{t=1}^{T} x_{t}^{\prime} V_{t} x_{t}+u_{t-1}^{\prime} P_{t-1} u_{t-1}\right\} .
$$

The mapping $f_{t}$ influences $\mathbf{E}\left\{J_{T}\right\}$ by producing $i_{t}$ based on $\mathbf{y}_{t-M_{t}}^{t}$ and $\mathbf{k}_{0}^{t-1}$. Hence, it influences the states and controls that depend on $i_{t}$, i.e., $\mathbf{x}_{t+1}^{T}$ and $\mathbf{u}_{t}^{T-1}$.

Let $\mathcal{S}_{i}\left(\mathbf{k}_{0}^{t-1}\right)$ denote the set of all $\mathbf{y}_{t-M_{t}}^{t}$ such that $i_{t}=i$ given $\mathbf{k}_{0}^{t-1}$. Since $\mathbf{E}\left\{J_{T}\right\}=\mathbf{E}\left\{\mathbf{E}\left\{J_{T} \mid \mathbf{y}_{t-M_{t}}^{t}, \mathbf{k}_{0}^{t-1}, i_{t}\right\}\right\}$, determining the optimal mapping $f_{t}\left(\mathbf{y}_{t-M_{t}}^{t}, \mathbf{k}_{0}^{t-1}\right)$ is equivalent to specifying the set $\mathcal{S}_{i}\left(\mathbf{k}_{0}^{t-1}\right)$ such that the inner term $c\left(\mathbf{y}_{t-M_{t}}^{t}, \mathbf{k}_{0}^{t-1}, i\right)=\mathbf{E}\left\{J_{T} \mid \mathbf{y}_{t-M_{t}}^{t}, \mathbf{k}_{0}^{t-1}, i_{t}=i\right\}$ is minimized over $i \in \mathcal{L}_{i}$ given $\mathbf{y}_{t-M_{t}}^{t}$ and $\mathbf{k}_{0}^{t-1}$. That is

$$
\begin{aligned}
\mathcal{S}_{i}\left(\mathbf{k}_{0}^{t-1}\right)=\left\{\mathbf{y}_{t-M_{t}}^{t}: c\left(\mathbf{y}_{t-M_{t}}^{t}, \mathbf{k}_{0}^{t-1}, i\right)\right. & \\
& \left.\leq c\left(\mathbf{y}_{t-M_{t}}^{t}, \mathbf{k}_{0}^{t-1}, i^{\prime}\right), 1 \leq i^{\prime} \leq L_{i}\right\} .
\end{aligned}
$$

Since for any $i, i^{\prime} \in \mathcal{L}_{i}$

$$
\begin{aligned}
& \mathbf{E}\left\{\sum_{s=1}^{t} x_{s}^{\prime} V_{s} x_{s}+u_{s-1}^{\prime} P_{s-1} u_{s-1} \mid \mathbf{y}_{t-M_{t}}^{t}, \mathbf{k}_{0}^{t-1}, i_{t}=i\right\} \\
& =\mathbf{E}\left\{\sum_{s=1}^{t} x_{s}^{\prime} V_{s} x_{s}+u_{s-1}^{\prime} P_{s-1} u_{s-1} \mid \mathbf{y}_{t-M_{t}}^{t}, \mathbf{k}_{0}^{t-1}, i_{t}=i^{\prime}\right\}
\end{aligned}
$$

because the choice of $i_{t}$ only influences "future" terms, we equivalently get

$$
\begin{aligned}
& \mathcal{S}_{i}\left(\mathbf{k}_{0}^{t-1}\right) \\
& =\left\{\mathbf{y}_{t-M_{t}}^{t}: c^{+}\left(\mathbf{y}_{t-M_{t}}^{t}, \mathbf{k}_{0}^{t-1}, i\right)\right. \\
& \left.\leq c^{+}\left(\mathbf{y}_{t-M_{t}}^{t}, \mathbf{k}_{0}^{t-1}, i^{\prime}\right), \quad 1 \leq i^{\prime} \leq L_{i}\right\},
\end{aligned}
$$

where

$$
\begin{aligned}
& c^{+}\left(\mathbf{y}_{t-M_{t}}^{t}, \mathbf{k}_{0}^{t-1}, i\right) \\
& =\mathbf{E}\left\{\sum_{s=t+1}^{T} x_{s}^{\prime} V_{s} x_{s}+u_{s-1}^{\prime} P_{s-1} u_{s-1} \mid \mathbf{y}_{t-M_{t}}^{t}, \mathbf{k}_{0}^{t-1}, i_{t}=i\right\} .
\end{aligned}
$$

This concludes the proof.

We refer to the sets $\mathcal{S}_{i}\left(\mathbf{k}_{0}^{t-1}\right)$ as the encoder regions. In general, for $M_{t}>1$, these are non-overlapping subsets of $\mathbb{R}^{\left(M_{t}+1\right) \times p}$. For $M_{t}=0$ and $p=1$ (scalar quantization), and assuming "smooth" (continuous or differentiable) pdf's, the regions are intervals or unions of intervals. In the Gaussian case (Gaussian initial-state and noise pdf's) our numerical results indicate that the regions always become intervals when the training is close to convergence. However, as it is possible to find controllers that result in disconnected optimal sets $\mathcal{S}_{i}\left(\mathbf{k}_{0}^{t-1}\right)$, it cannot be said in general that the encoder regions are intervals. This also means that it is not possible to parameterize the encoder regions as intervals without loss.

In Proposition 6 below, we present a similar result for the open-loop encoder in an open-loop encoder system. This result is useful when looking for locally optimal solutions to Problem 2 by iteratively alternating between optimizing the open-loop encoder and the CE controller.
Proposition 6: Consider a fixed controller $\mathbf{g}_{0}^{T-1}$ and the fixed open-loop encoder components $\overline{\mathbf{f}}_{0}^{t-1}, \overline{\mathbf{f}}_{t+1}^{T-1}$. Given the linear plant (1) and the memoryless channel (4), the open-loop encoder mapping $\bar{f}_{t}\left(\overline{\mathbf{y}}_{t-M_{t}}^{t}, \mathbf{j}_{0}^{t-1}\right)$ that minimizes the LQ cost (7) is given by

$$
\begin{aligned}
i_{t}= & \arg \min _{i \in \mathcal{L}_{i}} \mathbf{E}\left\{\sum _ { s = t + 1 } ^ { T } \left(x_{s}^{\prime} V_{s} x_{s}\right.\right. \\
& \left.\left.+u_{s-1}^{\prime} P_{s-1} u_{s-1}\right) \mid \overline{\mathbf{y}}_{t-M_{t}}^{t}, \mathbf{j}_{0}^{t-1}, i_{t}=i\right\} .
\end{aligned}
$$

The proof is similar to the one of Proposition 5 with the modification that $\left\{\overline{\mathbf{y}}_{t-M_{t}}^{t}, \mathbf{j}_{0}^{t-1}\right\}$ is the information available at the open-loop encoder. Since the CE controller in (16) is the optimum control strategy in an open-loop encoder system assuming a fixed open-loop encoder $\overline{\mathbf{f}}_{0}^{T-1}$, the following result is very useful.

Corollary 1: Consider an open-loop encoder system. There exists a solution to Problem 2 that satisfies the following conditions: The controller mapping is given by $u_{t}=\ell_{t} \hat{x}_{t}$ with $\ell_{t}$ as in (17) and $\hat{x}_{t}$ as in (6); and the open-loop encoder mapping $\bar{f}_{t}$ is given by

$$
i_{t}=\arg \min _{i \in \mathcal{L}_{i}} \mathbf{E}\left\{\sum_{s=t}^{T-1} \tilde{x}_{s}^{\prime} \pi_{T-s} \tilde{x}_{s} \mid \overline{\mathbf{y}}_{t-M_{t}}^{t}, \mathbf{j}_{0}^{t-1}, i_{t}=i\right\}
$$

where $\pi_{T-t}$ can be obtained by (19).

Proof: By Proposition 3 we know, given a fixed openloop encoder (in this case the optimal open-loop encoder), the CE controller (16) is the optimal control strategy. Also, it is clear from (19) that, the choice of $i_{t}$ influences only the term $\mathbf{E}\left\{\sum_{s=t}^{T-1} \tilde{x}_{s}^{\prime} \pi_{T-s} \tilde{x}_{s} \mid \mathbf{j}_{0}^{t}\right\}$ in cost-to-go $\gamma_{t+1}$. This concludes the proof.

In the case of full SI or the open-loop encoder system, the encoding rule (23) can be replaced with (24), in order to reduce computations. Also notice that the encoder in (24) directly minimizes the weighted estimation error

$$
\mathbf{E}\left\{\tilde{x}_{s}^{\prime} \pi_{T-s} \tilde{x}_{s}\right\}, s=t, \ldots, T-1 .
$$

Hence there is "separation" between quantization/coding/estimation and control, since the encoder tries to minimize the (weighted) average mean-squared error and since the CE controller splits into computing the estimate $\hat{x}_{t}$ and the scaling $\ell_{t}$. The encoding is influenced by the control problem only via the matrices $\pi_{T-s}, s=t, \ldots, T-1$, and computing $\hat{x}_{t}$ at the receiver side can be interpreted as decoding or estimation.

\section{A. Optimal Encoding for Some Special Linear Systems and Channels}

This subsection is devoted to a discussion of the encoder design in Proposition 5 for some special cases of the general system described in Section II-A. In particular, we compare the scenarios where process noise and measurement noise are absent and the communication link from the encoder to the controller is noiseless. The following results, stated without proof, follow from Proposition 5.

Consider a fixed controller $\mathbf{g}_{0}^{T-1}$, and the fixed encoder components $\mathbf{f}_{0}^{t-1}$ and $\mathbf{f}_{t+1}^{T-1}$, for a linear plant (1) and a memoryless channel (4). Then we arrive at the following: 
1) If $v_{t}=e_{t}=0$ and $j_{t}=i_{t}$, then $f_{t}\left(\mathbf{y}_{t-M_{t}}^{t}, \mathbf{k}_{0}^{t-1}\right)$ that minimizes the average LQ cost (7) is given by

$$
\begin{aligned}
i_{t}= & \arg \min _{i \in \mathcal{L}_{i}} \mathbf{E}\left\{\sum _ { s = t + 1 } ^ { T } \left(x_{s}^{\prime} V_{s} x_{s}\right.\right. \\
& \left.\left.+u_{s-1}^{\prime} P_{s-1} u_{s-1}\right) \mid \mathbf{x}_{t-M_{t}}^{t}, \mathbf{k}_{0}^{t-1}, i_{t}=i\right\}
\end{aligned}
$$

where the expectation is over the initial state distribution.

2) If $e_{t}=0$ and $j_{t}=i_{t}$, then $f_{t}\left(\mathbf{y}_{t-M_{t}}^{t}, \mathbf{k}_{0}^{t-1}\right)$ that minimizes the average LQ cost (7) is given by (25), where the expectation is over the initial state and the process noise distributions.

3) If $e_{t}=0$ and $j_{t} \neq i_{t}$, then $f_{t}\left(\mathbf{y}_{t-M_{t}}^{t}, \mathbf{k}_{0}^{t-1}\right)$ that minimizes the average LQ cost (7) is given by (25), where the expectation is over the initial state distribution, the process noise distribution and the channel distribution.

In all three cases, the optimal encoder is described by (25). However, the same expression leads to different levels of computational complexity. In the first case, the initial state is the only uncertainty involved in the estimation of the current controller state and the prediction of the future evolution. In the second case, the expectation takes not only the distribution of the initial state but also the distribution of the process noise into account. In the last case, the complexity is further increased, in order to additionally take transmission errors into consideration.

\section{Iterative EnCoder-Controller Design Algorithm}

In this section, we propose an encoder-controller design algorithm based on the results in Sections III and IV. The design is suitable in particular for low transmission rates, and since the algorithm strives to find good encoder-controller pairs for a given noisy channel, it introduces protection against transmission errors. That is, the result is a design for joint quantization, error protection and control. Since the controller information $\mathbf{j}_{0}^{t}$ has finite resolution, there are only a finite number of possible control commands. Thus, these values can be pre-calculated and stored in a codebook at the controller.

As mentioned, the overall joint encoder-controller optimization problem is typically not tractable, and we therefore propose to optimize the encoder-controller pair iteratively. There are two cases to handle separately:

1) Full SI: In this case, as discussed in Section III-B, we carry out the design for the open-loop encoder system in Fig. 3 and then use Proposition 2 to convert the solution to the original problem in Fig. 1.

2) Partial SI: In this case, we constrain the controller to be a CE controller, as discussed in Section III-C, and carry out the design for the original system in Fig. 1.

Fig. 4 depicts a flow-diagram of the design procedure, with notation for the original system (the case of partial SI). An initial encoder-controller pair is specified. Thereafter, each encoder-controller component, $f_{0}, g_{0}, \ldots, f_{T-1}, g_{T-1}$, is successively optimized. After one round, if the improvement is not below a pre-defined threshold $\delta$, a new round is started to update $f_{0}, g_{0}, \ldots, f_{T-1}, g_{T-1}$. At time $t$, first $f_{t}$ is updated for fixed $\mathbf{f}_{0}^{t-1}, \mathbf{f}_{t+1}^{T-1}, \mathbf{g}_{0}^{T-1}$ and then $g_{t}$ is updated for fixed $\mathbf{f}_{0}^{T-1}$, $\mathbf{g}_{0}^{t-1}, \mathbf{g}_{t+1}^{T-1}$. The currently derived $f_{t}$ will replace the former one to be regarded as a fixed component in optimizing the other components $\mathbf{f}_{0}^{t-1}, \mathbf{f}_{t+1}^{T-1}$ and $\mathbf{g}_{0}^{T-1}$, until next time when $f_{t}$ is

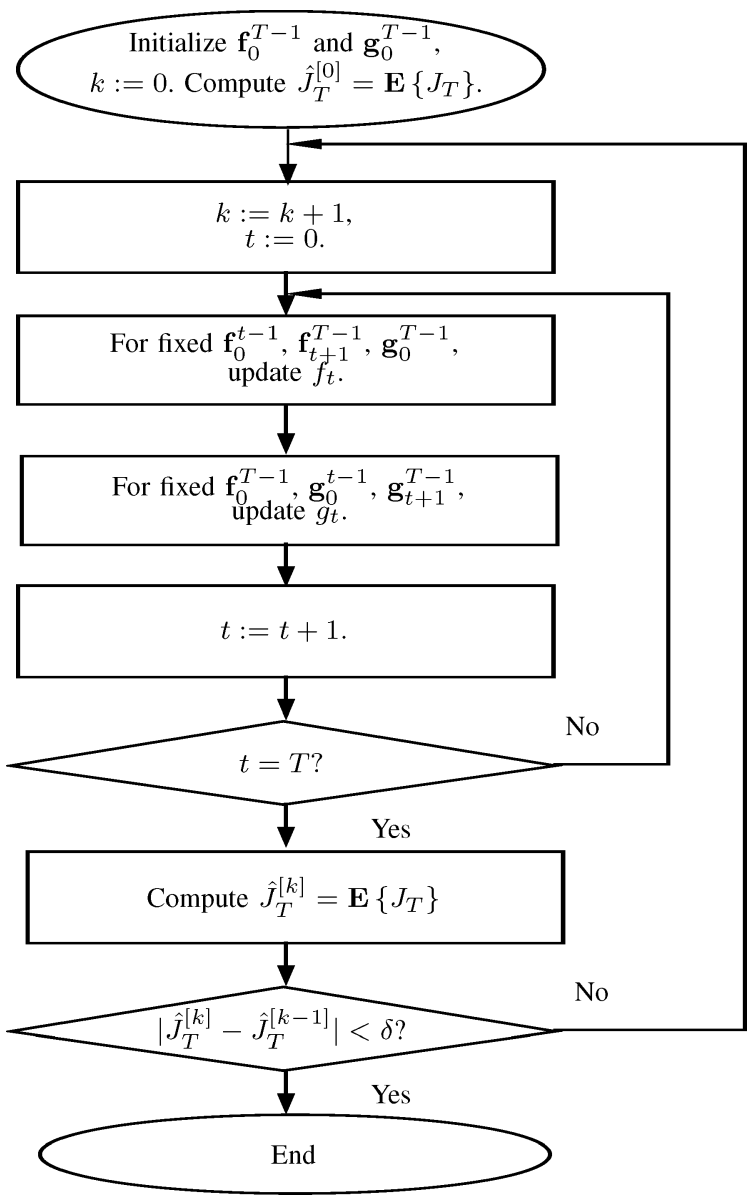

Fig. 4. Flow-diagram of the iterative encoder-controller optimization procedure. The variable $k$ is a counter for the number of rounds. In each round, all the encoder-controller components $\mathbf{f}_{0}^{T-1}$ and $\mathbf{g}_{0}^{T-1}$ are updated. The value $\hat{J}_{T}^{[k]}$ represents the resulting cost $\mathbf{E}\left\{J_{T}\right\}$ after round $k$. The iteration is terminated when the improvement in the system performance is less than a certain threshold $\delta$.

updated. The rules for updating the encoder-controller components are developed in Sections III and IV. In particular, we update the encoder component $f_{t}$ using (22) and the controller $g_{t}$ using (20)-(21).

In order to jointly optimize the open-loop encoder and controller in an open-loop encoder system, in the case of full SI, we use instead Proposition 3 to update the CE controller. For the encoding rule, we can use (23) or (24).

\section{A. On the Convergence of the Design}

In principle, the iterative design procedure always converges in the case of the open-loop encoder system. This is because the $\mathrm{CE}$ controller in Proposition 3 is optimal for any $\overline{\mathrm{f}}_{0}^{T-1}$. That is, each time Proposition 3 is invoked, given an updated encoder, the performance can only remain the same or improve. Similarly, each time the encoder is updated for a given controller, the resulting performance cannot decline. Hence, the design converges (since zero is a lower bound on the cost). This is the usual rationale behind proving convergence for iterative training-based designs for vector quantizers and related problems in which parts of a system are updated while others are held fixed [50], [51], [56]. Again, in principle, the design converges, and in this sense it reaches a local optimum. Still, nothing can in general be said about global optimality [56]. 
However, in the general case (with only partial SI) the design does not necessarily converge at all, since in the step where the controller is updated the expressions in (20)-(21) do not necessarily lead to a new controller with better performance, because the CE controller is not necessarily optimal for the given encoder. Still, in our numerical experiments this has not been a problem, and, empirically, the design algorithm appears to converge to a solution also in the general case. Loosely speaking, the explanation is that after a few iterations the fact that the new controller is a better $\mathrm{CE}$ controller than the previous one makes the performance improve (while during the first few iterations the performance need not improve in each step, depending on the initialization).

\section{B. Implementation and Numerical Considerations}

The encoder is specified by the encoder regions $\mathcal{S}_{i}\left(\mathbf{k}_{0}^{t-1}\right)$, $i \in \mathcal{L}_{i}, t=0, \ldots, T-1$. For $M_{t}>0$, it is difficult to parameterize these regions, as they are subsets of $\mathbb{R}^{\left(M_{t}+1\right) \times p}$. However, in the scalar case, $M_{t}=0$ and $p=1$, the regions can be specified by storing the boundaries between them. As mentioned in Section IV the regions are not necessarily intervals, however, according to our empirical experience they become intervals as the design converges. Hence, $\mathcal{S}_{i}\left(\mathbf{k}_{0}^{t-1}\right)$ can often be parameterized by storing at most $L_{i}-1$ real values for each possible $\mathbf{k}_{0}^{t-1}$. The controller, on the other hand, can always be specified by storing at most $L_{j}^{t+1}$ terms at time $t=0, \ldots, T-1$. Hence, while carrying out the design can be computationally intensive, the result can often be parameterized as a finite set of parameters. Thus, the proposed system, when designed, can in general be used at low or moderate complexity. Since the training can be carried out off-line, its complexity is not of crucial importance. Still, we comment on a few important aspects of the involved computations as follows.

Let us for example look at implementing the controller component $g_{t}(16)$. The linear feedback law $\ell_{t}$ is straightforward to compute, so the computational complexity is mainly determined by the calculation of the conditional mean estimate, $\mathbf{E}\left\{x_{t} \mid \mathbf{j}_{0}^{t}\right\}$. This entity needs to be re-calculated whenever any of other encoder-controller components $f_{s}, s \leq t$, and $g_{s}, s<t$, has been updated. Deriving an explicit expression for $\mathbf{E}\left\{x_{t} \mid \mathbf{j}_{0}^{t}\right\}$ is in general not possible. In our simulations, we therefore resort to using sequential Monte Carlo sampling to approximate the expressions involved in executing the design. Starting with a sample set for the $x_{0}$ 's, corresponding sample sets for $y_{0}, i_{0}, j_{0}, u_{0}$, $x_{1}$, etc., can be generated until a sample set that describes the conditional pdf $p\left(x_{t} \mid \mathbf{j}_{0}^{t}\right)$ is obtained and employed to estimate values for $\mathbf{E}\left\{x_{t} \mid \mathbf{j}_{0}^{t}\right\}$. The accuracy of the result is affected by the size of the sample sets. As the number of possible values for $\mathbf{E}\left\{x_{t} \mid \mathbf{j}_{0}^{t}\right\}$ increases exponentially with $T$, the training is not practical for problems with long horizon. Still, one approach to finding useful systems at longer horizon would be to design the system for, say, $T=4$ or 5 , and then keep the solution constant for a number of subsequent time-instants. The rationale being that an unknown $x_{0}$ usually would represent a triggering disturbance, that can be attenuated by the controller over a number of time-instants, while the remaining noise uncertainty can often be handled by a stationary solution.

For a more detailed discussion of implementation aspects we direct the reader to [44].
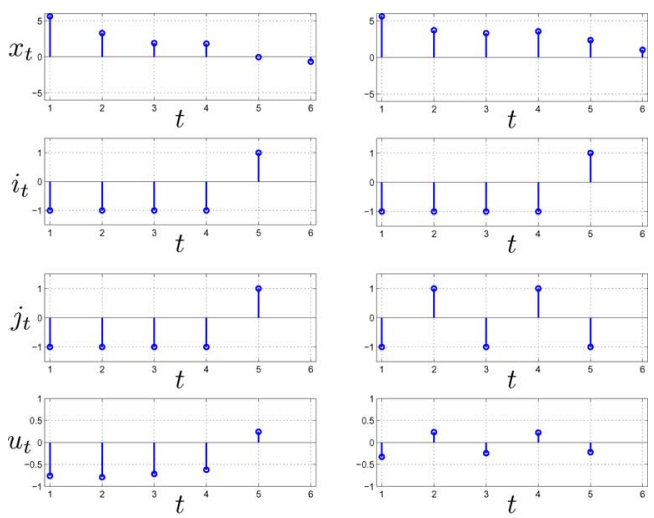

(a)

(b)

Fig. 5. System behavior is illustrated with respect to the crossover probability $\epsilon$. The state response $x_{t}$, the transmitted symbol $i_{t}$, the received symbol $j_{t}$ and the control $u_{t}$ are depicted. In this example, $\epsilon=0.04$ results in no transmission error and $\epsilon=0.3$ in three errors. (a) $\epsilon=0.04$. (b) $\epsilon=0.3$.

\section{NUMERICAL EXAMPLES}

Here we present numerical experiments to demonstrate the performance obtained by using the iterative encoder-controller design. We study the special case of the general system in Fig. 1 of a scalar linear system with measurements encoded and transmitted over a BSC. The system equations and the LQ cost are

$$
\begin{aligned}
x_{t+1} & =a x_{t}+u_{t}+v_{t}, \quad y_{t}=x_{t}+e_{t}, \\
J_{T} & =\sum_{t=1}^{T}\left(x_{t}^{2}+\rho u_{t-1}^{2}\right) .
\end{aligned}
$$

The BSC is described by the crossover probability $\epsilon=$ $P_{r}(0 \mid 1)=P_{r}(1 \mid 0)$. Let $c\left(i_{t}\right)$ be a binary codeword of length $R$ representing the encoder output $i_{t}$ for transmission via $R$ uses of the BSC. The mapping between $i_{t}$ and $c\left(i_{t}\right)$ is referred to as the index assignment. Likewise, $c\left(j_{t}\right)$ denotes the received $R$ bit codeword. Due to the independent transmission of each bit, the crossover probability $P_{r}\left(c\left(j_{t}\right) \mid c\left(i_{t}\right)\right)$ is

$$
P_{r}\left(c\left(j_{t}\right) \mid c\left(i_{t}\right)\right)=(1-\epsilon)^{R-d_{H}\left(c\left(i_{t}\right), c\left(j_{t}\right)\right)} \epsilon^{d_{H}\left(c\left(i_{t}\right), c\left(j_{t}\right)\right)}
$$

where $d_{H}\left(c\left(i_{t}\right), c\left(j_{t}\right)\right)$ is the Hamming distance between $c\left(i_{t}\right)$ and $c\left(j_{t}\right)$, i.e., the number of bits in which they differ.

In Fig. 5, the state response is depicted together with the transmitted symbol, the received symbol and the control. The system parameters are: $a=0.9, \rho=0.5, T=5$ and $R=1$. The initial state $x_{0}$, process noise $v_{t}$ and measurement noise $e_{t}$ are all zero-mean Gaussian distributed, with the variances $3,0.5$, and 0.5 , respectively. The system has been studied for the channel transition probabilities $\epsilon=0.04$ and 0.3 . It can be observed that the number of symbol errors increases with $\epsilon$. Since a symbol error can result in a control input doing more harm than good, as expected, the magnitude of the admissible control becomes smaller when the channel error increases.

In Fig. 6, we show the system performance in terms of the crossover probability $\epsilon$. The system parameters are: $a=1.1$, $\rho=0.5, T=2$ and $R=2$. The initial state $x_{0}$, process noise $v_{t}$ and measurement noise $e_{t}$ are all zero-mean Gaussian, with the variances $5,0.5$, and 0.5 , respectively. Performance $\bar{J}_{T}$ is obtained by normalizing $\mathbf{E}\left\{J_{T}\right\}$ with the expected cost obtained when no control action is taken, cf., the horizontal line in 


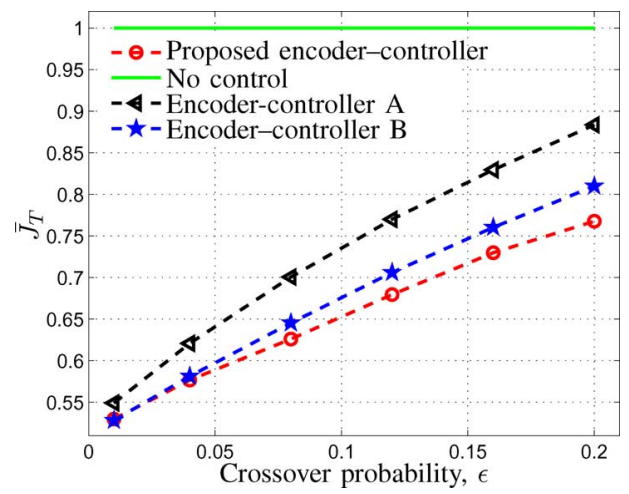

Fig. 6. Comparison of the control performance between the encoder-controller proposed in the paper and two heuristic encoder-controllers (encoder-controller A and encoder-controller B). Independent of the crossover probability, the proposed encoder-controller gives best performance.

Fig. 6. Three types of encoder-controller pairs are studied: our proposed encoder-controller is compared with two heuristic designs $\mathrm{A}$ and $\mathrm{B}$. The first pair, encoder-controller $\mathrm{A}$, is designed as follows. The measurement is quantized using a time-invariant uniform quantizer with the encoder thresholds $\{-2,0,2\}$ and the reconstructions $\{-3,-1,1,3\}$. At the controller, received indexes are mapped into reconstructions which are fed into a Kalman filter for estimating the state $x_{t}$. The Kalman filter is designed assuming the error due to measurement noise, quantization and transmission is white and Gaussian distributed. Thereafter, the control is calculated as a linear function of the Kalman filter output. The linear feedback law is $\ell_{t}$ in (17). By this method, the distortion due to quantization and channel error is treated as a part of measurement noise. Note that, the "extended" measurement noise (including quantization error and channel error) is in fact neither Gaussian nor uncorrelated with the state and the process noise, making the Kalman filter a sub-optimal estimator. The second pair, encoder-controller $\mathrm{B}$, utilizes a time-invariant uniform encoder with the encoder thresholds $\{-2,0,2\}$, together with the CE controller in (16). The last pair is an encoder-controller trained according to our proposed design in Section V where the encoder has full SI. It can be seen in the figure that the trained encoder-controller pair outperforms the other two schemes.

The side-information affects the system in several ways. In Fig. 7, we show a comparison of different degrees of SI when the encoder $\mathbf{f}_{0}^{T-1}$ is optimized. In particular, we explore no, incomplete and full SI scenarios. In the experiment, the same setting as in Fig. 6 is adopted. The incomplete SI is generated as follows. The least significant bit of the binary codeword $c\left(j_{t}\right)$ is discarded and the resulting codeword is fed back to the encoder over a noiseless link. We demonstrate the convergence properties of the training algorithm by showing the successive iteration results after each round.

The figure shows that full SI provides the best training result, while the incomplete SI scenario outperforms the no SI scenario. Generally in Fig. 7, the improvement given by knowing the SI is minor. One of the main reasons is that the trained encoder-controllers have resulted in similar densities $p\left(x_{t} \mid \mathbf{y}_{t-M_{t}}^{t}, \mathbf{k}_{0}^{t-1}\right)$, for different $\mathbf{k}_{0}^{t-1}$ 's. This can be partly explained by the experiment set-up, with a fairly short time horizon $(T=2)$, Gaussian distributed signals, and a CE controller. However, the advantage of having SI becomes more significant for longer time horizon,

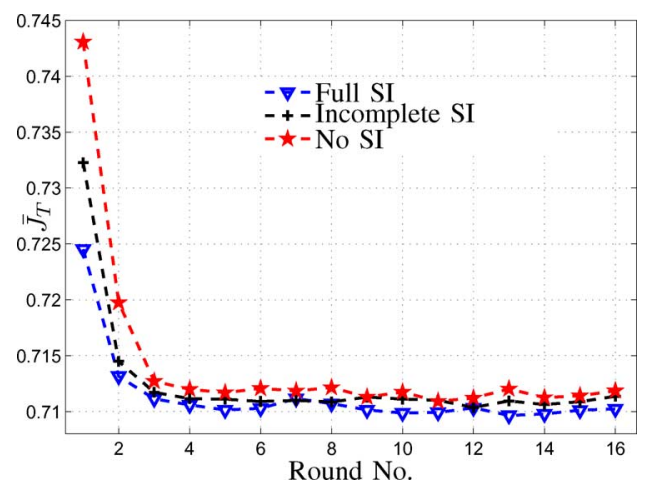

Fig. 7. Convergence of the training algorithm is illustrated with varying degree of SI, at $\epsilon=0.16$. Incomplete SI is generated by discarding the least significant bit of the received binary codeword $c\left(j_{t}\right)$ and feeding the new codeword back to the encoder over a noiseless channel.

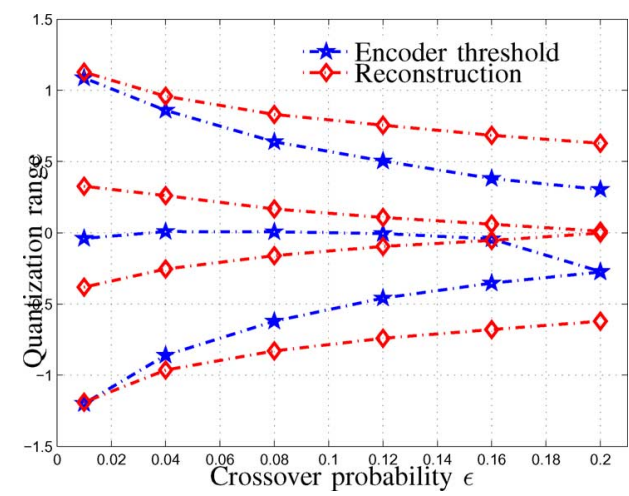

Fig. 8. Encoding boundaries given by $f_{0}$ and the corresponding reconstructions $\hat{x}_{0}$ with respect to $\epsilon$.

because SI can reduce the encoder uncertainty in the controller state which accumulates with time.

How the encoder and controller respond to increasing the channel noise is illustrated in Fig. 8, using the same experiment setting as in Fig. 6. In the figure, we demonstrate the partition of the real numbers defined by the encoder mapping $f_{0}$, and the corresponding reconstructions $\hat{x}_{0}$, for growing $\epsilon$. Recall that the control is a linear function of the reconstruction. We note that the number of controls chosen by the encoder decreases with increasing $\epsilon$. This phenomenon is well-known in quantization for noisy channels and is attributed to the varying abilities of binary codewords in combating channel errors. For very noisy channels, it is beneficial to transmit only the "stronger" codewords [51], providing true redundancy for error protection. Note that the asymmetry at $\epsilon=0.16$ is also a consequence of the fact that the binary codewords are unequally sensitive to the channel errors. Another impact of increasing $\epsilon$ is that the encoder thresholds and the controls are all moved closer to zero, indicating that only small-valued control actions are allowed.

\section{CONCLUSION}

This paper has investigated joint optimization of the encoder and the controller in closed-loop control of a linear plant with low-rate feedback over a memoryless noisy channel. We introduced an iterative approach to the design of encoder-controller pairs inspired by the traditional design of vector quantizers. In deriving design criteria, we arrived at new results regarding the optimality of certainty equivalence controllers in our setting. In the case of full SI, we introduced a "virtual help-system," the 
open-loop encoder system. We showed that a CE controller is optimal for any given encoder in this system, and we argued that encoder-controller pairs designed for the help-system can be translated to perform well in the original system. In the case of partial SI, we cannot claim that enforcing the CE controller structure is without loss. However, since the general controller problem is challenging in this case, we used CE controllers as sub-optimal, but practically feasible approximations.

We have performed various numerical investigations. Our results demonstrate the promising performance obtained by employing the proposed design algorithm. We also investigated the impact on system performance of various degree of SI at the encoder.

The present work focuses on a proof of concept, since the suggested nonlinear encoder-controller mappings are not practical when their memory is extended over time. Future work includes introducing structure in the encoder mapping, for example via graphical or trellis descriptions, to allow for extending the horizon in the cost criterion.

\section{ACKNOWLEDGMENT}

The authors would like to thank the associate editor and the anonymous reviewers for many very helpful comments that considerably helped to improve the quality of the paper.

\section{REFERENCES}

[1] D. F. Delchamps, "Stabilizing a linear system with quantized state feedback," IEEE Trans. Autom. Control, vol. 35, no. 8, pp. 916-924, Aug. 1990.

[2] W. S. Wong and R. W. Brockett, "Systems with finite communication bandwidth constraints - Part II: Stabilization with limited information feedback," IEEE Trans. Autom. Control, vol. 44, no. 5, pp. 1049-1053, May 1999.

[3] J. Baillieul, "Feedback coding for information-based control: Operating near the data-rate limit," in Proc. 41st IEEE Conf. Decision Control, Dec. 2002, vol. 1, pp. 3229-3236.

[4] B. Picasso, F. Gouaisbaut, and A. Bicchi, "Construction of invariant and attractive sets for quantized-input linear system," in Proc. 41th IEEE Conf. Decision Control, Dec. 2002, vol. 1, pp. 824-829.

[5] F. Fagnani and S. Zampieri, "Stability analysis and synthesis for scalar linear systems with a quantized feedback," IEEE Trans. Autom. Control, vol. 48, no. 9, pp. 1569-1584, Sep. 2003.

[6] F. Fagnani and S. Zampieri, "A symbolic approach to performance analysis of quantized feedback systems: The scalar case," SIAM J. Control Optim., vol. 44, no. 3, pp. 818-866, 2005.

[7] N. Elia and S. K. Mitter, "Quantization of linear systems," in Proc. 38th Conf. Decision Control, Dec. 1999, vol. 4, pp. 3428-3433.

[8] J. C. Delvenne, "An optimal quantized feedback strategy for scalar linear systems," IEEE Trans. Autom. Control, vol. 51, no. 2, pp. 293-303, Feb. 2006.

[9] R. W. Brockett and D. Liberzon, "Quantized feedback stabilization of linear systems," IEEE Trans. Autom. Control, vol. 45, no. 7, pp. 1279-1289, Jul. 2000.

[10] G. N. Nair and R. J. Evans, "Exponential stabilisability of finite-dimensional linear systems with limited data rates," Automatica, vol. 39, pp. 585-593, 2003.

[11] S. Tatikonda and S. Mitter, "Control under communication constraints," IEEE Trans. Autom. Control, vol. 49, no. 7, pp. 1056-1068, Jul. 2004.

[12] Q. Ling and M. D. Lemmon, "Optimal dynamic bit assignment in noise-free quantized linear control systems," in Proc. 44th IEEE Conf. Decision Control, Eur. Control Conf., Dec. 2005, pp. 8191-8196.

[13] D. Liberzon, "Stabilization by quantized state or output feedback: A hybrid control approach," in Proc. IFAC 15th Triennial World Congress, 2002.
[14] S. Tatikonda, A. Sahai, and S. Mitter, "Stochastic linear control over a communication channel," IEEE Trans. Autom. Control, vol. 49, no. 9 , pp. 1549-1561, Sep. 2004

[15] G. N. Nair and R. J. Evans, "Stabilizability of stochastic linear systems with finite feedback data rates," SIAM J. Contol . Opt., vol. 43, no. 2, pp. 413-436, 2004.

[16] G. N. Nair, R. J. Evans, I. M. Y. Mareels, and W. Moran, "Topological feedback entropy and nonlinear stabilization," IEEE Trans. Autom. Control, vol. 49, no. 9, pp. 1585-1597, Sep. 2004.

[17] A. V. Savkin, "Analysis and synthesis of networked control systems: Topological entropy, observability, robustness and optimal control," Automatica, vol. 42, pp. 51-62, 2006.

[18] G. N. Nair, F. Fagnani, S. Zampieri, and R. Evans, "Feedback control under data rate constraints: An overview," Proc. IEEE, vol. 95, no. 1, pp. 108-137, Jan. 2007.

[19] H. T. Simsek, "Anytime Capacity Coding with Feedback," Ph.D. dissertation, Univ. California, Berkeley, 2004.

[20] S. Tatikonda and S. Mitter, "Control over noisy channels," IEEE Trans. Autom. Control, vol. 49, no. 9, pp. 1196-1201, Sep. 2004.

[21] A. Mahajan and D. Teneketzis, "Fixed delay optimal joint source-channel coding for finite-memory systems," in Proc. IEEE Int. Symp. Inform. Theory, Jul. 2006, pp. 2319-2323.

[22] G. N. Nair and J. Baillieul, "Time to failure of quantized control via a binary symmetric channel," in Proc. 45th IEEE Conf. Decision Control, Dec. 2006, pp. 2883-2888

[23] A. Sahai and S. Mitter, "The necessity and sufficiency of anytime capacity for stabilization of a linear system over a noisy communication link: Part I: Scalar systems," IEEE Trans. Inform. Theory, vol. 52, no. 8, pp. 3369-3395, Aug. 2006.

[24] A. S. Matveev and A. V. Savkin, "An analogue of Shannon information theory for detection and stabilization via noisy discrete communication channels," SIAM J. Control Optm., vol. 46, no. 4, pp. 1323-1367, 2007.

[25] P. Minero, M. Franceschetti, S. Dey, and G. Nair, "Towards feedback stabilization over fading channels," in Proc. 5th Int. Symp. Modeling Optim. Mobile, Ad Hoc Wireless Networks Workshops, 2007, pp. 1-5.

[26] C. D. Charalambous and A. Farhadi, "LQG optimality and separation principle for general discrete time partially observed stochastic systems over finite capacity communication channels," Automatica, vol. 44, pp. 3181-3188, 2008.

[27] A. Gurt and G. N. Nair, "Internal stability of dynamically quantised control for stochastic scalar plants," Automatica, vol. 45, pp. 1387-1396, 2009.

[28] J. H. Braslavsky, R. H. Middleton, and J. S. Freudenberg, "Feedback stabilization over signal-to-noise ratio constrained channels," IEEE Trans. Autom. Control, vol. 52, no. 8, pp. 1391-1403, Aug. 2007.

[29] O. C. Imer, S. Yüksel, and T. Basar, "Optimal control of LTI systems over unreliable communication links," Automatica, vol. 42, pp. 1429-1439, 2006.

[30] E. I. Silva et al., "Simple coding for achieving mean square stability over bit-rate limited channels," in Proc. 47th IEEE Conf. Decision Control, Dec. 2008, pp. 2698-2703.

[31] S. Vembu, S. Verdu, and Y. Steinberg, "The source-channel separation theorem revisited," IEEE Trans. Inform. Theory, vol. 41, no. 1, pp. 44-54, Jan. 1995.

[32] A. Sahai, "The necessity and sufficiency of anytime capacity for control over a noisy communication link," in Proc. 43rd IEEE Conf. Decision Control, Dec. 2004, vol. 2, pp. 1896-1901.

[33] J. B. Lewis and J. T. Tou, "Optimum sampled-data systems with quantized control signals," IEEE Trans. Appl. Ind., vol. AI-82, no. 67, pp. 229-233, 1963.

[34] L. Meier, "Combined optimal control and estimation," in Proc. Allerton Conf. Circuit Syst. Theory, 1965, pp. 109-120.

[35] R. E. Larson, "Optimum quantization in dynamic systems," IEEE Trans. Autom. Control, vol. AC-12, no. 2, pp. 162-168, 1967.

[36] T. R. Fischer, "Optimal quantized control," IEEE Trans. Autom. Control, vol. AC-27, no. 4, pp. 996-998, Aug. 1982.

[37] A. S. Matveev and A. V. Savkin, "On a problem related to application of digital networked communication technology to stabilization of noisy plants over noisy channels," in Proc. IEEE Int. Conf. Control Appl., Oct. 2006, pp. 2072-2077.

[38] Q. Xu, "Anytime Capacity of the AWGN+Erasure Channel with Feedback," M.S. thesis, Univ. California, Berkeley, CA, 2005.

[39] V. S. Borkar and S. K. Mitter, "LQG Control with Communication Constraints," MIT, Cambridge, MA, Tech. Rep., 1995.

[40] M. Fu, "Linear quadratic Gaussian control with quantized feedback constraints," in Proc. IEEE Autom. Control Conf., Jun. 2009, pp. 2172-2177. 
[41] A. S. Matveev and A. V. Savkin, "An analogue of Shannon information theory for networked control systems: Stabilization via a noisy discrete channel," in Proc. 43nd IEEE Conf. Decision Control, Dec. 2004, vol. 4, pp. 4485-4490.

[42] V.S. Borkar, S. K. Mitter, and S. Tatikonda, "Optimal sequential vector quantization of Markov sources," SIAM J. Control Optim., vol. 40, no. 1 , pp. 135-148, 2001.

[43] A. S. Matveev and A. V. Savkin, "The problem of LQG optimal control via a limited capacity communication channel," Syst. Control lett., vol. 53, pp. 51-64, 2004.

[44] L. Bao, "Control Over Low-Rate Noisy Channels," Ph.D. dissertation, School Elect. Eng., Royal Institute of Technology, School of Electrical Engineering, Stockholm, Sweden, 2009.

[45] L. Bao, M. Skoglund, and K. H. Johansson, "Encoder-decoder design for event-trigged feedback control over bandlimited channels," in Proc. Amer. Control Conf., 2006, pp. 4183-4188.

[46] L. Bao, M. Skoglund, and K. H. Johansson, "Encoder-decoder design for feedback control over the binary symmetric channel," in Proc. IEEE Int. Symp. Inform. Theory, 2006, pp. 2481-2485.

[47] L. Bao, M. Skoglund, and K. H. Johansson, "On optimal system design for feedback control noisy channels," in Proc. IEEE Int. Symp. Inform. Theory, 2007, pp. 2486-2490.

[48] L. Bao, M. Skoglund, and K. H. Johansson, "A scheme for joint quantization, error protection and control over noisy channels," in Proc. Amer. Control Conf., 2007, pp. 4905-4910.

[49] T. M. Cover and J. A. Thomas, Elements of Information Theory. New York: Wiley, 1991.

[50] A. Gersho and R. M. Gray, Vector Quantization and Signal Compression. Norwell, MA: Kluwer, 1992.

[51] N. Farvardin, "A study of vector quantization for noisy channels," IEEE Trans. Inform. Theory, vol. 36, no. 4, pp. 799-809, Jul. 1990.

[52] M. Aoki, Optimization of Stochastic Systems - Topics in Discrete-Time Systems. New York: Academic Press, 1967.

[53] D. Bertsekas, Dynamic Programming and Stochastic Control. New York: Academic Press, 1976.

[54] H. S. Witsenhausen, "Separation of estimation and control for discrete time systems," Proc. IEEE, vol. 59, no. 11, pp. 1557-1566, Nov. 1971.

[55] Y. Bar-Shalom and E. Tse, "Dual effect, certainty equivalence, and separation in stochastic control," IEEE Trans. Autom. Control, vol. 19, no. 5, pp. 494-500, Oct. 1974.

[56] M. Sabin and R. Gray, "Global convergence and empirical consistency of the generalized Lloyd algorithm," IEEE Trans. Inform. Theory, vol. IT-32, no. 2, pp. 148-155, Mar. 1986.

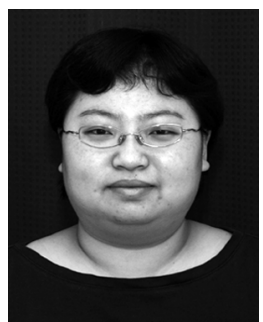

Lei Bao (S'04-M'09) received the M.Sc. degree in electrical engineering (with specialization in digital communication) from Chalmers university of Technology, Goteborg, Sweden, in 2003 and the Ph.D. degree in telecommunication from the Royal Institute of Technology (KTH), Stockholm, Sweden, in 2009.

She is currently working at the Communication Theory Lab, KTH. Her research interests include communication theory and coding theory for networked control

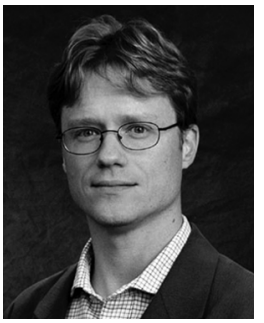

Mikael Skoglund (S'93-M'97-SM'04) received the Ph.D. degree from Chalmers University of Technology, Goteborg, Sweden, in 1997.

In 1997, he joined the Royal Institute of Technology (KTH), Stockholm, Sweden, where he was appointed Professor in 2003. At KTH, he heads the Communication Theory Lab and he is the Assistant Dean for Electrical Engineering. He has authored some 180 scientific papers, including papers that have received awards, invited conference presentations, and papers ranking as highly cited according to the ISI Essential Science Indicators. He has also consulted for industry, and he holds six patents. His research interests are in the theoretical aspects of wireless communications. He has worked on problems in source-channel coding, coding and transmission for wireless communications, Shannon theory and statistical signal processing.

Dr. Skoglund has served on multiple technical program committees for IEEE conferences. From 2003 to 2008, he was an Associate Editor with the IEEE TRANSACTIONS ON COMMUNICATIONS and he is presently on the editorial board for the IEEE TRANSACTIONS ON INFORMATION THEORY.

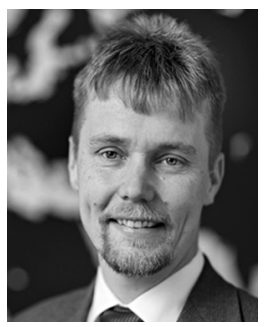

Karl Henrik Johansson (SM'08) received the M.Sc. and Ph.D. degrees in electrical engineering from Lund University, Lund, Sweden, in 1992 and 1997, respectively

$\mathrm{He}$ is Director of the ACCESS Linnaeus Centre and Professor at the School of Electrical Engineering, Royal Institute of Technology, Sweden. $\mathrm{He}$ is a Wallenberg Scholar and holds a Senior Researcher Position with the Swedish Research Council. He has held visiting positions at UC Berkeley (1998-2000) and California Institute of Technology (2006-07). His research interests are in networked control systems, hybrid and embedded control, and control applications in automotive, automation and communication systems.

Dr. Johansson received a six-year Individual Grant for the Advancement of Research Leaders from the Swedish Foundation for Strategic Research in 2005, the triennial Young Author Prize from IFAC in 1996, the Peccei Award from the International Institute of System Analysis, Austria, in 1993, and Young Researcher Awards from Scania in 1996 and from Ericsson in 1998 and 1999, respectively. He is the Chair of the International Federation of Automatic Control (IFAC) Technical Committee on Networked Systems since 2008. He has served on the IEEE Control Systems Society Board of Governors and on the Executive Committees of the European research projects HYCON and RUNES. He is on the editorial boards of the IEEE TRANSACTIONS ON AUTOMATIC CONTROL and the IET Control Theory \& Applications, and previously of the IFAC journal Automatica. 